\title{
Rhetorices contractae (1621), II, 24. De la consolation
}

Présentation, traduction et notes : Christine Noille

\section{G. J. Vossius}

Christine Noille (éd.)

\section{(2) OpenEdition}

Journals

Édition électronique

URL : http://journals.openedition.org/rhetorique/534

DOI : 10.4000/rhetorique.534

ISSN : 2270-6909

Éditeur

UGA Éditions/Université Grenoble Alpes

Édition imprimée

ISBN : 978-2-37747-010-5

\section{Référence électronique}

G. J. Vossius, «Rhetorices contractae (1621), II, 24. De la consolation », Exercices de rhétorique [En ligne], 9 | 2017, mis en ligne le 21 juin 2017, consulté le 10 décembre 2020. URL : http:// journals.openedition.org/rhetorique/534 ; DOI : https://doi.org/10.4000/rhetorique.534

Ce document a été généré automatiquement le 10 décembre 2020.

\section{(c) (i) (2)}

Les contenus de la revue Exercices de rhétorique sont mis à disposition selon les termes de la Licence Creative Commons Attribution - Pas d'Utilisation Commerciale - Partage dans les Mêmes Conditions 4.0 International. 


\section{Rhetorices contractae (1621), II, 24. De la consolation}

Présentation, traduction et notes : Christine Noille

\section{G. J. Vossius}

Christine Noille (éd.)

\section{Présentation. Rituel de la consolation : Vossius et ses prédécesseurs}

1 Nous poursuivons l'édition des chapitres que Vossius consacre aux types de discours, édition commencée dans l'Atelier d'Exercices de rhétorique 1 | 2013'.

2 La rhétorique pédagogique et pratique définit le genre de la consolation par un rituel que l'on peut synthétiser en quatre points :

- dresser un monument oratoire sur la douleur, en faisant de la consolation une véritable pièce d'éloquence et en lui donnant l'ampleur, le decorum et l'ornatus adaptés aux différentes circonstances: pour la liste des cas distingués par Vossius, voir ci-dessous le plan du chapitre II, 24 ;

- travailler sur la malevolentia de l'âme en peine, sur son rejet a priori de la parole consolatoire et son refus de toute remédiation (ou purgation) : voir ci-dessous la recension des raisons de la captatio benevolentiae chez Vossius ;

- rendre l'affligé plus fort, en accumulant les arguments éthiques (appel à la vertu, à la sagesse) et les arguments pathétiques (mettant en jeu l'espoir et l'assurance, la honte et l'émulation) : voir ci-dessous la recension des raisons du réconfort chez Vossius; et infra note 101 , pour une synthèse.

- ritualiser la parole consolatoire par la reprise polyphonique des voix anciennes (poètes, orateurs et philosophes) : voir ci-dessous la recension des exemples et des citations chez Vossius et ses prédécesseurs.

3 Nous avons choisi d'éditer avec les Rhetorices contractae [...] libri quinque de Vossius (1621) les deux références majeures que celui-ci utilise et synthétise dans son chapitre 
sur la consolation : d'abord le chapitre III, 122 des Poetices libri septem de Scaliger (Les sept livres de la Poétique, 1561), qu'il cite littéralement à deux reprises; et les chapitres 49 et $50 \mathrm{du}$ De Conscribendis epistolis d'Érasme (De la manière d'écrire des lettres, 1522), qu'il paraphrase constamment. Nous nous sommes efforcés de traduire de la même façon d'un texte à l'autre les expressions latines qu'ils avaient en commun. Pour le protocole éditorial voir ci-dessous « Édition».

\section{Vossius, Rhetorices contractae, II, 24 : le plan du chapitre}

Nota bene : la numération des paragraphes est de Vossius ; pour résumer leur contenu, nous ajoutons ici les intitulés.

$\S 1$-2. Définition de la consolation

§ 3. Division : malheur avéré (§ 4-31) / malheur à venir (§ 32-33)

$\S 4$. Cas des malheurs avérés : deux divisions, selon la qualité du malheur (§ 5-7) et selon les personnes (§ 8-14)

$\S 5-7$. Cas des malheurs avérés auxquels on peut remédier : consolations plaisantes (§ 6) - consolations centrées sur les conjectures (§ 7)

§ 8- 31 : Cas des malheurs avérés et graves

$\S 8$. Dans l'exorde : les raisons de consoler (réglage de l'éthos)

§ 9-31 : subdivision suivant la personne de celui qui est consolé : consolation pour les âmes fortes ( $\$ 10)$ et consolation pour les âmes faibles (\$ 11-31: deux méthodes)

$\S 10$. Consolation pour les âmes fortes : par renforcement (exorde, argumentaire, péroraison)

$\S 11-24$. Consolation pour les âmes faibles : $\mathbf{1}^{\text {ère }}$ méthode

$\S 11-14$. Dans l'exorde : captatio benevolentiae par transfert de la douleur sur soi (d'où amor, affection) et amplification du malheur d'autrui (d'où : pitié)

§15-23. L'argumentaire: les raisons du réconfort par incitation à la décision (stratégie délibérative selon les lieux de l'honorable, l'utile, le nécessaire, l'agréable, le possible)

$\S 24$. La péroraison : exhortation

$\S 25-31$. Consolation pour les âmes faibles : $2^{\mathrm{e}}$ méthode

§ 15-26. Dans l'exorde: les raisons de consoler (réglage de l'éthos) et la captatio benevolentiae (d'où : affection et pitié)

§ 27-31: L'argumentaire: les raisons du réconfort par conversion éthique (itinéraire en cinq stations: l'humaine condition, la vertu virile, la sagesse philosophique, la foi chrétienne, l'exemplarité chrétienne)

$\S 32-33$. Cas des malheurs à venir (l'exemple des consolations politiques)

\section{Vossius, Rhetorices contractae, II, 24 : les raisons de la captatio benevolentiae}

Par ordre d'apparition :
A. La plaisanterie
B. Le devoir de mise en garde
C. L'affection
D. Au nom des autorités
E. Le devoir d'amitié
F. L'éloge de l'affligé pour sa constance
G. La grandeur du mal (le mal ou malheur qui est enduré)
H. Notre propre douleur
I. La parenté 


\section{Vossius, Rhetorices contractae, II, 24 : les raisons du réconfort}

Par ordre d'apparition :

1. La conjecture

2. La nécessité de souffrir et de mourir

3. La bonne conscience

4. La patience face aux épreuves (le courage de les supporter)

5. Les suites heureuses

6. Les malheurs plus grands

7. La Providence (la volonté de Dieu)

8. La réputation accrue par le malheur

9. La bonification morale par l'épreuve

10. L'irritation du mal par les pleurs

11. Le souvenir futur du malheur présent

12. Les exemples de constance

13. La fermeté virile

14. La sagesse prudentielle

15. Le mépris du monde

16. La dépossession de soi

17. La vie après la mort

18. Le devoir d'exemplarité

\section{Les exemples chez Vossius et ses prédécesseurs}

- Présence des orateurs dans les chapitres 49 et 50 du De conscribendis epistolis d'Érasme (1522) Florilèges d'arguments consolatoires extraits des lettres de Cicéron, de Pline le Jeune et d'Érasme lui-même, à la suite des remarques générales (op. cit., ch. 49) ainsi que le florilège pour les réponses (ibid., 50). Édition avec traduction dans le présent numéro, section ATELIER.

Référence à des lettres intégrales :

Cicéron, Familiares, 4.3 [consolation à Servius Sulpicius sur les calamités publiques]

Cicéron, Familiares, 4.5 [consolation de Servius «Sulpicius » ou Sixticius à Cicéron sur la mort de sa fille Tullia]

Cicéron, Familiares, 4.6 [réponse à la consolation de Servius Sulpicius sur la mort de sa fille]

Cicéron, Familiares, 4.8 [consolation à Marcellus sur les vicissitudes politiques]

Cicéron, Familiares, 5.13 [réponse à la consolation de Lucceius]

Cicéron, Familiares, 5.16 [consolation à Titius sur la mort d'un de ses enfants]

Cicéron, Familiares, 5.18 [consolation à T. Fadius sur sa disgrâce]

Cicéron, Familiares, 6.1 [consolation à Aulus Torquatus sur son bannissement]

Cicéron, Familiares, 6.6 [consolation à Cécina sur son exil]

Cicéron, Familiares, 9.16 [consolation plaisante à Papirius Paetus sur ses difficultés financières]

Cicéron, Lettres à Brutus, 1.9 [consolation (sur la perte d'un proche ?)]

Modèles de lettres forgées par Érasme (voir notre édition dans la section ATELIER) :

Consolation à un proche avec objurgation

Consolation plaisante à un proche

Réponse à une lettre de consolation

Consolation à Antoine Sucquet sur la mort de son fils (non traduit)

Consolation à Canidius sur son exil (non traduit)

- Présence de la poésie : vers cités par Vossius (1621) et Érasme (1522)

Aulu-Gelle, Nuits attiques, 17.14, «Choix des meilleures pensées des comédies de Publius [Mimus]»

Horace, Odes, 1.24.19-20

Lucrèce, De la nature des choses, 3.971 
Stace, Silves, 2.1, Chant funèbre pour Glaucias Melior

Térence, Andrienne, 2.1.9

Virgile, Les Bucoliques, 1.6

Virgile, L'Énéide, 6.95-96.

- Référence à des corpus consolatoires chez Vossius (1621) et Scaliger (1561)

Aelius Aristide, Discours funèbre en l'honneur d'Étéonée (Orationes, 31)

Anonyme (attribué à Ovide), Consolation à Livie Augusta sur la mort de Drusus Néron son

fils (9 av. J. C. -2 ap. J.C.)

Cicéron, Lettres familières

Plutarque, Consolation à Apollonius [sur la mort de son fils]

Plutarque, Consolation à sa femme [sur la mort de leur fille]

Sénèque, Consolation à ma mère Helvie [sur l'exil de son fils]

Sénèque, Consolation à Marcia [sur la mort de son fils]

Sénèque, Consolation à Polybe [sur la mort de son frère]

\section{Principes d'édition}

\section{Édition}

Édition princeps : Rhetorices contractae, sive Partitionum Oratoriarum libri quinque, Leyde, Joannes Maire, 1621 (que nous n'avons pu voir ; exemplaire à la B. M. de Dijon, cote 6248). Soit, en français : La Rhétorique abrégée, ou les Divisions de l'art oratoire, en cinq livres; le mot Rhetorices est le génitif singulier du grec rhètorikè.

Nous donnons le texte de la $3^{\mathrm{e}}$ édition chez le même Joannes Maire, 1640 (exemplaire de la B. M. de Lyon, visible sur Google Livres), p. 200-210. La mention d'une « $3^{e}$ édition » était déjà au titre de l'édition Maire de 1627, et le sera encore au titre de l'édition Maire de 1650. D'où, dans la page de titre de notre édition Maire de 1640, le long paragraphe qui suit la mention « Editio tertia recognita, \& aucta ». Il explique qu'est ensuite donné le même texte qu'une édition « toute récente » dont le texte était « bien plus correct que les éditions précédentes, et augmenté pour quelques passages de la main même de l'auteur »; une courte liste d'errata est donnée peu après, en vis-à-vis de l'épître dédicatoire. Cette insistance sur la correction du texte s'explique par la destination scolaire de cette édition de 1640, commande officielle : la page de titre précise en effet « Ex decreto Illustr. ac Pot. HollandiaE \& WeSTFRISAE DD. ORDINUM in usum Scholarum ejusdem Provinciae excusi. » En tout état de cause, l'édition de 1640 est la dernière édition chez Maire avant la mort de Vossius en 1649 (l'édition Maire suivante est celle de 1650).

Texte corrigé sur collation des éditions de : Iéna, J. Birckner, 1648 ; Leipzig, Christian Kirchner, 1660 ; Saumur, R. Péan, 1677 ; Leipzig, M. G. Weidmann, 1742 ; Madrid, Antonio de Sancha, 1781. Principales modifications au texte de l'éd. Maire 1640 : nous remplaçons transferamus par transferemus ( $\$ 11$ ) et fuisse jure censetur par fuisse censetur ( $\$ 19$, doublon avec le Iure en tête de phrase). En revanche, nous maintenons doleamus au $\$ 26$, même si le dolemus de l'éd. Kirchner 1660 paraît plus satisfaisant.

Nous reprenons le paragraphage et les numéros de sections tels qu'établis par Vossius. Nous rajoutons entre crochets des titres intermédiaires. Les brèves notes 
de Vossius (ou de son éditeur) sont données en notes, entre guillemets et précédées de la mention « Note de Vossius ».

Dans le latin, nous supprimons les accents et les lettres liées ; résolvons les abréviations ; translittérons le grec en caractères romains ; ajoutons les guillemets et indiquons entre crochets droits les pages de l'édition Maire de 1640. En vue de la recherche automatisée par mots, nous dissimilons le $i$ du $j$ et le $u$ du $v$; et nous régularisons des emplois très variables, en ramenant par exemple quum à cum, qvam à quam, caussa à causa.

\section{De la consolation / De consolatione}

\section{$\S 1$}

7 Nous en venons à la troisième catégorie [du genre délibératif ${ }^{2}$, à savoir les discours ${ }^{3}$ qui se consacrent à la douleur d'autrui ${ }^{4}$, tels que sont les discours de consolation ${ }^{5}$.

Pervenimus ad tertiam classem, cujus orationes versantur circa dolorem alienum : ut sunt consolatoriae.

\section{$\S 2$}

8 La consolation est un discours par lequel nous tranquillisons l'âme de celui qui est affligé .

CONSOLATIO est oratio, qua animum moerentis reddimus tranquillum.

\section{$\S 3$}

Or, la douleur qui doit être dissipée provient, soit de la perte d'un bien, soit de la crainte de le perdre ${ }^{7}$.

Dolor vero, qui abstergendus est, vel provenit ex bono jam amisso, vel ex amittendi metu.

\section{§ 4 [Cas des malheurs avérés]}

10 La consolation dont le propos est d'apaiser une douleur du premier genre est construite de différentes manières, selon la nature des maux qui nous frappent ou de la personne : celle qui console et celle qui a besoin de consolation ${ }^{8}$.

[p. 201] Consolatio, cui prioris generis dolorem lenire propositum est, diversimode instituitur, pro diversa natura, vel mali, quod obtigit; vel personae; sive ejus, quae consolatur ; sive illius, cui consolatione opus est.

\section{$\S 5$}

11 C'est dans la chose même qu'il faut voir si les maux sont légers, ou du moins tels qu'on puisse y remédier ${ }^{9}$.

In re ipsa videndum; num malum leve sit, vel tale saltem, quod corrigi possit. 


\section{$\S 6$}

12 S'ils sont légers, il sera permis d'ajouter des plaisanteries, pourvu que celui à qui nous nous adressons puisse les supporter ${ }^{10}$.

Si leve sit, licebit jocos admiscere, dum is ferre eos possit, quem compellamus.

\section{$\S 7$}

13 S'ils sont tels qu'on puisse y remédier, nous alléguerons des conjectures ${ }^{11}$, d'où il apparaîtra avec vraisemblance que ces maux ne dureront pas longtemps ${ }^{12}$.

Si corrigi possit, conjecturas adferemus, unde verisimile fiat, malum hoc non fore diuturnum.

\section{$\S 8$ [Cas des malheurs avérés et graves]}

14 Celui qui tient un discours de consolation est soit un inférieur, soit un égal, soit un supérieur ${ }^{13}$. Si c'est un inférieur, il doit agir avec plus de circonspection. Il témoignera avant tout de son attachement, et, pour ne pas paraître tirer son savoir de lui-même plus que d'autrui, il affirmera que ces paroles, qu'il est nécessaire de dire ${ }^{14}$, il les a un jour entendues d'un aïeul, d'un philosophe ou d'un personnage d'une sagesse éminente. Si c'est un égal, il dira qu'il agit selon les lois communes de l'amitié. Le supérieur sera plus libre que les deux précédents: si l'on montre une douleur excessive, il pourra même solennellement en faire le reproche.

15 Voir à ce sujet Scaliger au chapitre 123 du livre III de sa Poétique ${ }^{15}$ : "Celui qui tient un discours de consolation, dit-il, est supérieur, inférieur ou égal. Je comprends la grandeur en termes de pouvoir, de dignité, de richesses, de sagesse ou d'âge. Autre en effet sera la consolation d'Ovide par Livie, autre celle de Livie par Ovide ${ }^{16}$. Cela tient au pouvoir. Mais il peut y avoir aussi une différence de rang, indépendamment du pouvoir. Comme, par exemple, si un père s'adresse à son fils, Pompée à Cicéron ${ }^{17}$. Une différence de richesse : par exemple si Crassus s'adresse à un de ses clients issu de la plèbe ${ }^{18}$. Une différence de sagesse, comme Sénèque quand il s'adresse à Polybe ou à sa mère ${ }^{19}$. Pour l'âge, il n'est pas besoin d'exemples. Le supérieur fera intervenir son autorité, voire ira jusqu'aux reproches ${ }^{20}$. Le sage ajoutera aussi une argumentation en pro et contra; les maximes seront chez lui plus abondantes ${ }^{21}$. L'inférieur montrera de l'affection et dira que ce qu'il avance, il le tient d'hommes sages ${ }^{22}$. L'égal témoignera de son amitié, des lois communes du dévouement ${ }^{23}$.»

Consolator vel minor est, vel aequalis, vel major. Minori agendum circumspectius. Amorem suum imprimis testabitur: et, ne de suo sapere plus altero velle videatur, ea, quae dictu opus, ex parente grandaevo, vel quopiam philosopho, vel alio summae prudentiae viro, se aliquando audiisse profitebitur. Aequalis pro communi se amicitiae jure facere dicet. Major utrisque aget liberius, adeo ut, siquis immoderatius dolorem ferat, etiam increpet graviter. [p. 202] Scite hac de re Scaliger lib. III. de re poët. cap. cXXIII. "Consolator, inquit, aut est major, aut minor, aut aequalis. Magnitudinem intelligo, aut imperio, aut dignitate, aut opibus, aut sapientia, aut aetate. Aliter enim consolabitur Nasonem Livia, aliter Liviam Naso. Hoc spectat ad imperium. Dignitas autem etiam sine imperio esse potest. Veluti si pater filium, Ciceronem Pompeius. Opibus, ut si e plebe quempiam clientem suum Crassus. Sapientia, quemadmodum Polybium aut matrem Seneca. De aetate non opus est exemplis. Major interponet auctoritatem, etiam objurgabit. Sapiens apponet etiam disputationem. Sententiae huic crebriores. Minor ostendet affectum: sese id a sapientibus accepisse. Aequalis testabitur amicitiam : jus commune benevolentiae. " 


\section{$\S 9$} consolation ou bien a une âme trop faible pour admettre un adoucissement, surtout si sa douleur est récente ${ }^{25}$.

Is, qui consolandus est, vel videri vult fortior, quam ut egeat consolatione: vel animo est infirmiori, quam ut, recenti adeo dolore, solatium admittat.

\section{$\S 10$ [Consolation pour les âmes fortes]}

Dans le premier $\operatorname{cas}^{26}$, nous dirons que, bien qu'il s'agisse là d'un malheur qui pourrait entamer la constance de n'importe qui d'autre, cependant, sa sagesse et son exceptionnelle force d'âme nous sont apparues pleinement; par conséquent, nous n'en doutons pas, ces malheurs ne briseront pas du tout son âme $^{27}$. Car il est depuis longtemps arrivé, grâce aux préceptes salutaires de la philosophie, au point de savoir que c'est une loi commune aux mortels que de devoir supporter l'adversité ${ }^{28}$; et que le sage ne prend sur lui que la faute et qu'on ne peut être blessé que par soi-même ${ }^{29}$. Enfin nous le féliciterons ${ }^{30}$ pour cette grandeur d'âme ${ }^{31}$.

Prius si fuerit, dicemus, quamquam is casus est, qui alium quemvis de constantiae gradu dejicere posset, tamen ejus sapientiam, et eximiam fortitudinem, ita nobis esse perspectam, ut non dubitemus, quin in istis malis animo sit futurus plane infracto. Quippe qui salutaribus philosophiae praeceptis hoc jamdiu fuerit consecutus, ut sciat, hanc communem esse mortalium legem, ut pati necesse [p. 203] sit multa adversa: et sapientis nihil esse praestare praeter culpam : neque laedi quemquam posse, nisi a se ipso. Denique magnitudini huic animi gratulabimur.

\section{$\S 11$ [Consolation pour les âmes faibles : 1 ère méthode]}

Si au contraire son âme est faible ${ }^{32}$, et sa douleur si récente qu'elle semble rejeter tout remède, le discours sera en deux parties ${ }^{33}$. Dans la première ${ }^{34}$, nous transférerons sur nous-mêmes l'état d'âme de ceux qui souffrent, pour paraître plutôt céder à notre propre douleur que vouloir adoucir celle d'autrui.

Sin animus est infirmus, et dolor ita recens, ut respuere videatur medicinam ; bipartita erit oratio. Priori parte animum dolentium in nos transferemus, ut nostro magis dolori obsequi, quam alienum mitigare velle, videamur.

\section{$\S 12$}

Nous affirmerons que nous sommes peu aptes à consoler les autres, puisque notre âme est si déchirée que nous avons nous-mêmes besoin de consolation.

Affirmabimus, nos parum esse idoneos, qui solemur alios, cum tantopere animi discruciemur, ut consolatione ipsi egeamus.

\section{$\S 13$}

Et, pour que notre interlocuteur croie que nous parlons du fond du cœur, nous produirons les raisons de notre douleur, à savoir : la gravité du mal qui l'affecte et l'injustice dont il souffre ; et à cet endroit, on glissera même son éloge $e^{35}$. 
Atque, ut ex animo hoc dicere credamur, causas doloris nostri afferemus : nempe gravitatem mali, quo quis est affectus; et indignitatem perpetientis; ubi et laus ejus admiscetur.

\section{$\S 14$}

21 Nous ajouterons que nous ne sommes pas les seuls à souffrir pour ces raisons : c'est le cas de tous les gens de bien ${ }^{36}$.

22 Telle sera la première partie, dont la fonction est de persuader plus facilement autrui de supporter l'adversité d'une âme grande et forte. Pour cela, il faut qu'autrui voie que nous sommes très affectés par son sort si triste, mais aussi que nous le supportons d'une âme égale ${ }^{37}$. À l'inverse, ceux qui ne sont pas émus par nos maux, nous les haïssons en retour ${ }^{38}$ : rien n'est donc plus nuisible pour persuader.

Addemus, non nos modo propterea dolere, sed omnes bonos.

Et talis erit prima pars. Qua consequimur hoc, ut facilius persuadeamus alteri, adversam fortunam magno fortique esse animo ferendam: quippe qui videat, multum quidem affici nos casu tam tristi : verumtamen aequo eum animo tolerare. At illos, qui non commoventur [p. 204] malis nostris, etiam odimus : quo nihil ad persuadendum noxium est magis.

\section{$\S 15$}

Dans la deuxième partie du discours, nous avancerons les arguments propres à apaiser la douleur ${ }^{39}$ : comme affirmer que, si le mal est grave, avec de la patience on peut l'adoucir ${ }^{40}$.

24 Selon le mot du poète de Venouse :

«Cela est dur! Mais la patience allège

ce qui ne peut être changé ${ }^{41}$. »

Altera vero orationis parte adferemus ea, quae idonea sunt dolori leniendo. Quale fuerit, si

affirmes, malum grave quidem esse; sed quod patientia possit mitigari.

Secundum illud Venusini poetae:

«Durum, sed levius fit patientia

Quicquid corrigere est nefas.»

\section{$\S 16$}

Ou nous dirons au contraire qu'il n'est pas aussi grave qu'il y paraît. Car il ne durera pas longtemps. Ou qu'il peut être compensé par un bien autre. Ou que bien d'autres en supportent de plus graves ${ }^{42}$.

Imo nec grave adeo esse, quam videtur. Nam non futurum hoc diuturnum. Vel, bono alio posse resarciri. Vel, graviora multo alios perpeti.

\section{$\S 17$}

Nous tirerons ici avant tout ${ }^{43}$ nos arguments des lieux de l'honorable, de l'utile, du nécessaire, de l'agréable, du possible ${ }^{44}$.

Argumenta hic imprimis sumemus ab honesto, utili, necessario, jucundo, possibili. 


\section{$\S 18$}

De l'honorable : c'est la volonté divine, la raison l'ordonne, la nature de la vertu l'exige ; ainsi, nous adoucirons la douleur de son âme par la conscience d'avoir agi de façon juste ${ }^{45}$.

Ab honesto, quia haec divina est voluntas, hoc ratio dictat, hoc virtutis exigit natura, ut animi dolorem recte factorum conscientia mitigemus.

\section{$\S 19$}

\section{$\S 20$}

Du nécessaire : ce qu'on ne peut fuir, les pleurs ne le corrigent pas mais l'exaspèrent ${ }^{48}$.

D'où, comme le dit Publius Mimus :

"Supporte, ne blâme pas ce qui ne peut être évitét9."

[p. 205] A necessario, quia, quae effugere non licet, ea lugendo non corrigimus, sed exasperamus.

Eoque, ut ait Publius Mimus :

«Feras, non culpes, quod vitari non potest."

\section{$\S 21$}

De l'agréable : quelque ingrat que soit le sort présent, le temps viendra où le souvenir des malheurs passés fera naître le plaisir, quand ce souvenir est associé à une réputation honorable ${ }^{50}$.

A jucundo, quia, utcunque nunc ingrata sors ista sit; tamen veniet tempus, quo voluptatem

gignet praeteritorum malorum memoria cum honesto nomine conjuncta.

\section{$\S 22$}

Du possible : il n'est misère si grande qu'on ne puisse vaincre par la patience ${ }^{51}$.

A possibili, quia nulla est calamitas tanta, quin patiendo vincatur.

\section{$\S 23$}

On ajoutera beaucoup de force à ce dernier argument en utilisant les exemples ${ }^{52}$ éclatants de ceux qui ont supporté l'adversité avec constance. Et si ce sont des exemples de notre pays, ils toucheront encore bien plus $^{53}$. 
Ac postremo isti argumento multum roboris accesserit ab illustribus eorum exemplis, qui adversa constanter pertulere. Quae si domestica fuerint, multo etiam amplius movebunt.

\section{$\S 24$} et tel que nous-mêmes n'en sommes pas médiocrement affligés ; mais nous dirons qu'il ne faut pas le juger si dur qu'on souffre qu'il affaiblisse et brise l'âme de son poids ${ }^{60}$.

Deinde agemus de natura mali. Quod fatebimur grave quidem esse, et tale, ut propterea ipsi etiam doleamus non mediocriter: sed negabimus, durum adeo judicandum, ut ejus molestia quisquam animum suum debilitari ac frangi patiatur.

\section{$\S 27$}

Après cela, nous tirerons quatre sortes d'arguments de la personne qui endure ce malheur ${ }^{61}$. D'abord nous commencerons par une mise en garde, en l'engageant à songer qu'il est homme, et qu'il doit donc supporter la condition humaine avec constance ${ }^{62}$.

41 Par exemple, à qui pleure sans mesure la mort d'un mari ou d'une épouse ${ }^{63}$, nous affirmerons que ce fut, que c'est, que ce sera le sort partagé par tous que de quitter cette vie tôt ou tard ; et qu'il n'y avait pas de raison qu'il ou elle échappât à la loi qui est commune à tous les hommes ${ }^{64}$, et, bien plus, à tout ce qui naît, puisque tout ce qui est né doit mourir ${ }^{65}$. Stace dit avec élégance :

«...Tu ne vois partout que mort passée ou à venir : les nuits courent au néant, et les jours et les astres; et la terre même, malgré sa masse solide, n'y échappera pas. Les peuples, la race des mortels, les morts de la plèbe éphémère, qui les pleure ? Les uns, la guerre les réclame; les autres, c'est la mer. Ceux-ci trouvent la mort dans l'amour, ceux-là dans la folie cruelle de l'ambition. Tairai-je les fléaux ? L'aigre souffle de l'hiver 
pour les uns, le mortel Sirius et son feu implacable pour les autres, ou bien le pâle Automne et sa pluvieuse haleine. Tout ce qui a commencement doit craindre d'avoir fin ; tous nous irons, nous irons ${ }^{66}$. $»$

Post illa ex persona ejus, quae malum patitur, quadrifariam argumentabimur. Primum monebimus, ut cogitet, hominem sese, eoque humanam conditionem ferre constanter oportere.

Ut, sicui immoderate lugeatur mors mariti, vel uxoris, affirmabimus, hanc fuisse, esse, fore sortem omnium, ut serius, ocius, vita haec deseratur; nec causam fuisse, cur ille, illave eximeretur communi hominum legi, atque adeo omnium, quae ortum habent; quando omnia, quae ejusmodi sunt, occidunt. Pulcre Statius Papinius : «Omnia functa

Aut moritura vides. obeunt noctesque diesque Astraque, nec solidis prodest sua machina terris. Nam populos, mortale genus, plebisque caducae Quis fleat interitus? Hos bella, hos aequora poscunt; [p. 207] His amor exitio, furor his, et saeva cupido. Ut sileam morbos; hos ora rigentia brumae, Illos implacido letalis Sirius igni, Hos manet imbrifero pallens auctumnus hiatu. Quicquid habet ortus, finem timet. Ibimus omnes, Ibimus.»

\section{$\S 28$}

On en viendra ensuite à l'argument de l'homme viril ${ }^{67}:$ il ne s'agit pas seulement d'appartenir à la condition humaine, mais d'être un mâle. Il ne doit donc pas avoir une âme faible et efféminée.

Cicéron, lettre 11 du livre $\mathrm{V}^{68}$ : « C'est de vous rappeler que vous êtes homme, et homme de courage; que comme tel vous devez vous résigner aux chances communes de l'humanité, et supporter en sage ce qu'il n'était au pouvoir de personne de prévenir ou de détourner ${ }^{69}$.

Vraiment, qu'y a-t-il de plus honteux ${ }^{70}$, pour ceux qui se veulent hommes, que de montrer un cœur de femme? Et pourtant, dans les pleurs du deuil ${ }^{71}$, rien de plus répandu. Quand le législateur des Lyciens s'en rendit compte, il voulut que les hommes qui pleurent pour le deuil s'habillent en femmes. Il signifiait par là que les pleurs lors du deuil étaient plus dignes d'une femme que d'un homme qui a reçu une éducation libérale; si bien aussi que, sous un tel accoutrement, l'âme retrouvait plus vite la tranquillité. C'est ce que dit Plutarque dans la Consolation à Apollonius ${ }^{72}$.

Huic in viro succedet quod non homo solum sit, sed etiam mas : eoque non debeat esse animo molli, et effeminato.

Cicero epist. XI. lib. V. "Rogaremque, ut et hominem te, et virum esse meminisses : id est, communemque ${ }^{73}$, incertumque casum, quem nec vitare quisquam nostrum, neque praestare ullo pacto potest, sapienter ferres. "

Sane quid turpius, quam ut, viri qui esse volunt, animos gerant muliebres? Et tamen in luctu nihil pervulgatum magis. Quod cum videret Lyciorum legislator; voluit, ut mares lugentes femineo uterentur habitu. Quo significarat, luctum esse quid muliere magis dignum, quam viro liberaliter instituto: tum etiam, ut schemate tali animus ad tranquillitatem citius reduceretur. Auctor Plutarchus Consolatione ad Apollonium. 


\section{$\S 29$} chrétienne. Or on devient chrétien à une condition : être mis à l'épreuve en subissant les désastres de ce siècle, afin de ne pas mourir avec le monde ${ }^{80}$. Ici on pourra également emprunter au lieu commun sur le rôle de la providence divine ${ }^{81}$ dans nos malheurs ${ }^{82}$.

Quartum, nobis quidem Christi Sospitatoris cultoribus, illud est, quod non modo quis civili est prudentia instructus; sed Christiana etiam religione sit imbutus. Atqui ea Christiani conditione facti sunt, ut calamitatibus hujus seculi exerceantur, ne cum mundo pereant. Delibari quoque hic poterit locus communis de providentia divina in malo.

\section{$\S 31$}

Si celui auquel on s'adresse n'est pas seuleme
d'âmes $^{83}$, on en tirera le cinquième argument.

À savoir : qu'il n'appartient pas seulement à l'humanité mais qu'il est un homme viril ; qu'il n'est pas seulement un homme viril, mais qu'il possède en outre une sagesse qui le recommande ; et non seulement cela, mais en même temps qu'il est un chrétien, et qui plus est un chrétien qui doit offrir aux autres chrétiens des exemples de patience et de force d'âme, pour éviter de détruire d'un côté ce que l'on bâtit de l'autre ${ }^{84}$. On connaît cette maxime de Denys Caton :

« Il est honteux ${ }^{85}$ pour un censeur d'être repris pour ce que lui-même a blâmé ${ }^{86}$. »

Voilà pour la consolation du premier genre ${ }^{87}$. Si l'on veut y exceller, deux auteurs éminents, parmi les modernes, seront utiles : Érasme, dans son traité intitulé De la manière d'écrire des lettres, et Jules Scaliger au livre III de sa Poétique, chap. 123. Parmi les Anciens, d'une part les rhéteurs, et parmi eux, Ménandre, livre II, chapitre VIII ; d'autre part, les orateurs et les philosophes, comme Cicéron dans tant de lettres; et en outre Servius Sulpicius pour sa lettre à Cicéron sur la mort de Tullia; les lettres de Sénèque à Polybe, à Marcia, à Helvia ; Plutarque à Apollonius et à sa femme ; Aristide aux parents d'Étéonée ${ }^{88}$, etc. 
Quod siquis non Christianus tantummodo sit, sed etiam doctor Christianus : jam quintum inde petetur argumentum.

Ut qui non homo duntaxat sit, sed etiam vir; nec vir modo, sed insuper prudentia commendabilis; nec talis modo, sed simul Christianus, atque adeo, quem Christianis aliis patientiae et fortitudinis exempla praeire oporteat; ne quod una manu exstruit, id altera destruat. Notum est illud Dionysii Catonis :

"Turpe est doctori, cum culpa redarguit ipsum. »

Atque haec dicta sunto de consolatione primi generis: in qua praestare aliquid si voles, $e$ recentioribus quidem juvabunt duo summi viri ; Erasmus commentatione illa, quam [p. 209] inscripsit de epistolis conscribendis; ac Iulius Scaliger lib. III. de re poetica cap. CXXIII : e veteribus autem, cum Rhetores, et hos inter Menander lib. II. cap. VIII; tum oratores et philosophi, ut Tullius tot epistolis; ac praeterea Servius Sulpitius epistola ad Ciceronem de morte Tulliae; item Seneca ad Polybium, Marciam, Helviam; Plutarchus ad Apollonium, et uxorem; Aristides ad Etonei parentes, atque alii.

\section{$\S 32$ [Cas des malheurs à venir]}

51 Le deuxième genre console ceux qu'afflige la menace d'un malheur qu'ils jugent imminent ${ }^{89}$.

Par exemple, si quelqu'un vient à être soupçonné de conjuration par un tyran ${ }^{90}$. Comment il conviendrait de traiter une consolation de cette sorte, je ne puis l'exprimer mieux que Jules Scaliger. Voici ce qu'écrit ce grand homme ${ }^{91}$ :

Alterum genus solatur moerentes, eo quod jam malum sibi putent imminere.

Ut siquis apud tyrannum venerit in suspicionem conjurationis. Quomodo hujusmodi consolationem tractari conveniat, non melius possum dicere, quam Iulii Scaligeri verbis. Sic vero scribit summus vir.

\section{$\S 33$}

«Une telle consolation devra être divisée en deux parties ${ }^{92}$. Dans la première, on dira l'espoir, de pouvoir soit détruire la suspicion, soit apaiser la colère ${ }^{93}$. Dans la seconde, on renforcera le courage, on méprisera l'événement, on montrera que ce que nous estimons le plus est ce qui a le moins de valeur ${ }^{94}$. Enfin, il faudra emprunter aux philosophes et à ceux qui sont les contempteurs du monde ${ }^{95}$ et de la vie tout ce qu'ils disent sur le sujet. Et si leurs préceptes, sentences et proverbes sont éculés, il faudra les exprimer avec de tout autres mots, rythmes ${ }^{96}$ et figures. Car rien n'émeut moins que les choses familières, connues, quotidiennes ${ }^{97}$. Si au contraire ceux qu'on cherche à consoler ont la plus grande horreur des opinions et pratiques du vulgaire, et que, pour cette raison, ils sont en état d'affronter leur détresse ${ }^{98}$, il ne faudra pas tant arracher leur conviction ${ }^{99}$ par des raisonnements et des arguments qu'insinuer la persuasion par l'usage [répété] d'exemples ${ }^{100}$. » En voilà assez pour la consolation ${ }^{101}$.

"Consolatio talis in duas partes erit dividenda. In prima pones spem, qua vel opinio deleri, vel ira placari possit. In secunda addes animos, eventum contemnes, carissima quaeque ostendes esse vilissima: denique a philosophis, mundi vitaeque contemptoribus, omnia mutuanda. Quae si vulgata sint praecepta, sententiae, proverbia, aliis omnino verbis, numeris, figuris, exprimenda sunt. Nihil enim [p. 210] minus movet quam familiaria, nota, atque cotidiana. Sin a vulgi opinionibus usuque maxime abhorrent, ac propterea aegre animum subire valent : tum vero non tam rationibus, atque argumentis, fides extorquenda, quam exemplorum persuasione insinuanda. » De consolatione satis. 


\section{NOTES}

1. URL : http://rhetorique.revues.org/165. Sur les types ou genres du discours, voir Fr. Goyet, «Le problème de la typologie des discours», Exercices de rhétorique 1|2013, URL: http:// rhetorique.revues.org/122; et $\mathrm{Ch}$. Noille, «Les genres du discours dans l'ancienne rhétorique : listes, schémas et mode d'emploi, avec un exemple (le discours de Germanicus) », Exercices de rhétorique, 3 | 2014, URL : http://rhetorique.revues.org/337.

2. Dans ses Rhetorices contractae..., Vossius divise en quatre catégories ou «classes » les discours délibératifs, en prenant comme critère général les materia (les sujets, ce sur quoi ils portent). Plus précisément, à l'intérieur des materia, ce sont les passions qui dans le délibératif vont opérer le classement : première classe, les discours qui jouent sur les raisons de la colère, de la haine ou de l'amour (discours de mise en garde ou de recommandation, de sédition ou d'union: voir Rhetorices contractae..., op. cit., II, 21) ; deuxième classe, les discours qui jouent sur les raisons de l'intrépidité ou de la crainte (exhortations à faire ou à ne pas faire : voir ibid., II, 23) ; troisième classe, les discours visant à agir sur la douleur (les consolations : ibid., II, 24); quatrième classe, les discours qui jouent sur les raisons de la gratitude (les requêtes : voir ibid., II, 25). Sur la liste des passions en rhétorique, voir Aristote, Rhétorique, 2.1-11, trad. P. Chiron, Paris, Flammarion, «GF », 2007, p. 259-327. Pour leur adaptation par Vossius, voir Rhetorices contractae..., op. cit., II, $1-14$.

3. Une pratique oratoire orale de la consolation, sous forme de discours (oratio), est attestée chez deux rhéteurs que pratique Vossius : Ménandre le rhéteur (ou Ménandre de Laodicée : fin du III ${ }^{\mathrm{e}}$ siècle de notre ère) et Bartholomaüs Keckermann (c. 1572 - 1608, enseigne l'hébreu à Heidelberg puis est recteur de l'université de Danzig). Cf. Ménandre, 2.9, "Du discours de consolation", dernier paragraphe (éd. et trad. angl. D. A. Russell and N. G. Wilson dans Menander rhetor, Oxford, Clarendon Press, 1981, p. 161-165 ; trad. H. Caffiaux, dans H. Caffiaux, De L'Oraison funèbre dans la Grèce païenne, Valenciennes, Lemaître, 1864, p. 276-278). Et dans B. Keckermann, Systema rhetoricae, $1^{\text {re }}$ éd. 1606 (Hanovre, Wilhelm Antonius, 1608, $2^{\mathrm{e}}$ part. Rhetoricae specialis, livre I, p. 678), le paragraphe $\S 8$ consacré à l'actio des consolations (« Actio pauca et moderata sit [...]» «L'action doit être limitée et mesurée [...]»).

Ce rappel s'impose dans la mesure où les modèles dominants de la consolatio ne sont pas des discours, des orationes au sens circonstancié de pièces d'éloquence destinées à être prononcées, mais la lettre et le poème. C'est ainsi que les deux grandes références doctrinales mobilisées dans le présent chapitre sont les remarques d'Érasme sur la lettre consolatoire (De conscribendis epistolis, $1^{\mathrm{e}}$ éd. Bâle, Froben, 1522, chap.49-50, abrégé en De conscribendis..., 49-50: pour sa traduction, voir dans le présent numéro la section ATELIER) et celles de Scaliger sur les poèmes et plus précisément sur le genre des silves (Scaliger distingue onze genres poétiques dont les silves, elles-mêmes subdivisées, dans vingt-deux chapitres, en une quarantaine de catégories dont la dernière est la consolation: Poetices libri septem, $1^{\mathrm{e}}$ éd. Lyon, Antoine Vincent, 1561, III, 123, ultérieurement corrigé en III, 122, que nous abrégerons en Poetices..., III, 122 : pour sa traduction, voir dans le présent numéro la section ATELIER). Pour les lettres consolatoires, les exemples d'Érasme viennent de Cicéron, Pline le Jeune, Sénèque, Plutarque, mais aussi de lettres qu'il invente ad hoc; pour les silves de consolation, Scaliger a en tête les poèmes de Stace. Un seul exemple de discours en prose est mentionné ici par Vossius (et encore n'est-ce pas tout à fait une consolation : voir infra, note 88) : le «Discours à l'occasion des funérailles d'Étéonée » d'Aelius Aristide (Or. 31).

Comment comprendre que le terme oratio puisse, chez Vossius, recouvrir des formes aussi éloignées du discours que les lettres ou les poèmes ? C'est que la rhétorique de l'argumentation, telle que Vossius l'illustre, se situe à un point de vue d'où lettres, pièces d'éloquences et poèmes 
de consolation peuvent être tenus pour équivalents : à savoir leur commune visée argumentative, leur contentio, pour reprendre le terme de Cicéron désignant la tension qui unit un ensemble d'énoncés vers une fin unique, à savoir le dessein persuasif ou propositio (sur la contentio, voir De officiis 1.132 ; ibid., 2.48 ; id., Orator, 64 ; id., Tusculanae, 2.56-57). Pièces d'éloquence, lettres et poésies dont la propositio est de consoler sont donc tendus vers cette fin : en résultent pour elles toutes une même cohérence vectorisée, un même plan dynamique, un même dispositif argumentatif qu'analyse le présent chapitre. C'est ce dispositif argumentatif cohérent et dynamique qui définit dans un sens technique l'oratio et qui s'applique à toutes ces diverses formes. Sur ce point, voir Ch. Noille, «Les genres du discours à l'épreuve des lettres de fiction. Le cas de la première portugaise " dans C. Lignereux dir., Les rituels épistolaires, Paris, Garnier, à paraitre.

4. Vossius spécifiant chaque genre oratoire du délibératif par telle ou telle passion, quelles passions sont agitées dans le cadre de la consolation? Rien n'est synthétisé dans les Rhetorices contractae... En revanche, dans ses Institutiones (Commentariorum rhetoricorum, sive oratoriarum institutionum libri sex, $1^{\mathrm{e}}$ éd. Leyde, Van der Aa, 1605 : éd. utilisée Leyde, J. Maire 1630, I, 4, III, dernier alinéa, p. 31-32 ; abrégé en Institutiones), Vossius était plus explicite. Il définissait tout d'abord la suasoire délibérative par sa capacité à émouvoir, c'est-à-dire à mouvoir les passions (affectus); ce qui lui permettait de présenter ainsi la consolation (ibid., p. 30) : «Nam [...] consolatoria sit affectuosa », "Car [...] la consolation doit mettre en jeu les passions ». Et il énumérait ensuite les passions sur lesquelles argumente la consolation: amour, pitié, espoir, honte, émulation.

Contemporain des Institutiones de Vossius, le traité de Bartholomaüs Keckermann (Systema rhetoricae, op.cit., $2^{\mathrm{e}}$ part., livre I, chap. 10-20, p. 619-739) définit un système complexe de hiérarchisation des passions : à l'intérieur des « orationes affectuosae » (des discours qui jouent sur les affects), la consolation (ibid., chap.15, p. 675-678) correspond (au même titre que les exhortations et les requêtes) au cas i) des discours qui intègrent à titre de composantes parmi d'autres des séquences pathétiques (ibid., chap. 14, p. 660); ii) des discours qui visent à adoucir (ibid., chap. 15, p. 667) ; et iii) des discours mixtes (qui intègrent plusieurs passions : ibid., p. 661) : en l'occurrence, pour la consolation, l'assurance, l'espoir, l'émulation, la honte, la crainte.

Sur Keckermann et Vossius, voir T. M. Conley, Rhetoric in the European Tradition, University of Chicago Press, 1990, p. 157 sq. Sur une synthèse des passions évoquées au fil du présent chapitre, voir infra, note 101 .

5. La consolation n'épuise pas tous les discours « qui se consacrent à la douleur d'autrui ». Ce que semble désigner ici Vossius, c'est la nébuleuse compassionnelle formée par les divers discours de circonstance prononcés à l'occasion des cérémonies du deuil: oraisons funèbres chez les chrétiens (qu'elles soient prononcées au moment de l'enterrement ou ultérieurement), monologues funèbres, épicèdes, nénies, offrandes mortuaires et parentalia chez les païens (dont les différences tiennent essentiellement à leur place dans le rituel). Sauf que ces genres ne relèvent pas du délibératif, mais du démonstratif: ils consacrent certes une partie de leur développement à l'amplification du malheur et à la déploration; mais la majeure partie de leur argumentaire est dévolu à l'éloge (du défunt); et ils font généralement peu de place à l'adoucissement de la douleur et au réconfort. Chez Vossius, la consolation garde dans certains cas en mineure les séquences de déploration (exorde); elle admet le cas échéant la possibilité d'une séquence d'éloge (exorde) ; mais elle amplifie systématiquement les séquences de réconfort (argumentaire) et peut même accueillir une séquence d'exhortation (péroraison), laquelle l'enracine du côté du délibératif.

6. Voir Scaliger, Poetices..., III, 122, phrase incipitiale : « La consolation est un discours qui rend sa tranquillité à l'âme de celui qui est affligé. » C'est le thème stoïcien de la tranquillité de l'âme, voir par exemple le De tranquillitate animi de Sénèque. 
7. Voir Scaliger, Poetices..., III, 122, phrases introduisant le dernier mouvement du chapitre : «Voilà pour la consolation à l'occasion d'une perte. Si celui à qui on s'adresse est dans la crainte que survienne un événement néfaste [...].» Vossius commence ici une série de distinctions complexes, parce qu'héritées de classements différents. Il se souvient à la fois de la tripartition d'Érasme (1. consolation directe et franche; 2. consolation oblique et précautionneuse; 3. consolation plaisante), dont il retient les deux dernières catégories; et de la bipartition de Scaliger selon la persona, la personne de l'orateur (c'est-à-dire selon sa situation hiérarchique par rapport à son destinataire) et selon le malheur lui-même (avec une subdivision temporelle : selon que la perte est avérée ou à venir).

D'où un système qui chez Vossius est à la fois démultiplié et disproportionné : soit le malheur est avéré (§ 4-31) soit il menace ( $\$ 32-33$, principalement les risques politiques); et dans le premier cas, on peut à nouveau distinguer entre les différents malheurs, selon qu'ils semblent légers et réversibles (\$ 5-7, ils « ne dureront pas longtemps ») et irréversibles (revers politiques et deuils : $\S 8-32$ ). Vossius introduit ensuite d'autres subdivisions, par la personne du locuteur (selon son éthos, §8: infériorité, supériorité, égalité; souvenir de Scaliger) et par la personne du destinataire (fierté : § 9-10; ou abattement: §11-14; souvenir d'Érasme). Les divisions selon l'éthos du locuteur n'introduisent que des variantes dans l'exorde (au niveau des « rationes dicendi ", des raisons qui obligent à parler), tandis que les variations selon la personne du destinataire, pertinentes uniquement dans les cas de malheur avéré et grave, entraînent deux canevas consolatoires différents.

À partir de là, nous retiendrons qu'il y a pour Vossius quatre grandes classes de consolation (de développement visant à adoucir un malheur) : la consolation plaisante (simplement mentionnée : souvenir d'Érasme) ; la consolation " pour le mal [...] qu'on craint » (associée au revers politique : souvenir de Scaliger) ; et en situation focale, les deux types de consolation « pour le mal qu'[on] a reçu ». Les deux formules françaises viennent de J. du Roure, La Rhétorique française, Paris, chez l'auteur, 1672, p. 76, graphies modernisées (« On console quelqu'un ou du mal qu'il a reçu, ou de celui qu'il craint. ») : traité rédigé à partir des Rhetorices contractae... de Vossius, en particulier en ce qui concerne le développement sur la consolation, ibid., p. 76-77, intéressant pour les exemples français qu'il ajoute. Dans ses Institutiones (III, 4), Vossius avait recours à une division encore plus thématique : non pas selon les modalités du malheur (avéré ou non, léger ou grave, et dans ce dernier cas, selon la persona de l'affligé) ; mais selon les objets de la perte : d'abord le deuil, puis la consolation aux "aveugles, aux sourd et aux muets", la consolation aux infirmes (goutte, claudication, amputation...), la consolation en cas d'exil, la consolation en cas de banqueroute, la consolation en cas d'indignité.

8. Distinction reprise de Scaliger. Voir Poetices..., III, 122, la séquence transitionnelle : « Voilà pour les personnes. En ce qui concerne ce qui est arrivé, on doit en tirer différents arguments [...]. »

9. Voir deux remarques espacées dans Érasme, De conscribendis..., 49: "Nous atténuerons le malheur si nous montrons qu'il sera de courte durée. [...] Mais si l'infortune semble du genre à devoir durer, nous userons d'autres façons de procéder pour l'atténuer. " Et ibid. : "Dans la consolation la plaisanterie sera absolument à proscrire, à moins qu'il ne s'agisse d'une légère infortune, et dans ce cas on pourra le dérider avec une plaisanterie. » Vossius va ici amalgamer les deux cas (§ suivant).

10. Voir Érasme, De conscribendis..., 49 : «Si nous écrivons ainsi [i.e. en plaisantant], pourvu que celui à qui nous écrivons soit du genre à être charmé par de telles choses, il faudra systématiquement [...]. » Érasme fait ainsi le lien entre plaisanterie et captatio benevolentiae (« [...] du genre à être charmé par de telles choses »). C'est la première occurrence, dans le protocole consolatoire, d'une stratégie de captation de «bienveillance » (ou "affection »), qu'il convient d'interpréter à la lumière de la rhétorique de l'exorde (voir Cicéron, De inventione, 1.15-18.20-26). Capter les bonnes dispositions de l'auditeur (sa benevolentia), son attention (attentio) et s'assurer de sa bonne compréhension (docilitas) peuvent s'opérer selon deux voies : la voie directe et brève 
si l'auditeur est amical, attentif et intelligent; la voie indirecte et longue (ou insinuatio) si par aventure l'auditeur n'a ni envie ni loisir ni capacité de comprendre. Or la douleur place d'emblée de ce côté-là, en rendant l'auditeur sourd et rétif à la consolation : avant que de réconforter, il importe donc d'opérer la "purgatio malevolentiae », la purgation d'un auditeur mal disposé à écouter. C'est ainsi qu'à la suite d'Érasme, Vossius consacre un certain nombre d'analyses aux raisons de la captatio benevolentiae, autrement dit aux arguments préparatoires visant à instaurer les conditions d'audibilité et d'acceptation pour l'argumentaire du réconfort proprement dit.

Et parmi ces raisons est donc mentionnée ici, dans le cas très particulier des infortunes légères, le recours à la plaisanterie (argument $\mathrm{A}$ ). Nous recenserons par une numérotation alphabétique chaque nouvel argument visant à la captatio préalable de l'âme affligée. Pour leur récapitulation, voir supra, "Présentation». Pour le recours à la plaisanterie dans le dispositif exordial, voir Cicéron, De inventione, 1.17.25.

11. Argument des conjectures $\left(n^{\circ} 1\right)$, qu'Érasme renvoie à « l'état de cause conjectural ». C'est le premier argument consolatoire proprement dit que Vossius mentionne en substance. Nous recenserons par une numérotation avec chiffres arabes chaque nouvel argument. Pour leur récapitulation, voir supra, "Présentation » et infra, note 101. Les arguments consolatoires visent à réconforter et sont par conséquent distincts des arguments qui visent au préalable à capter les bonnes dispositions de l'âme affligée : voir infra note 39. Sur les conjectures dans les consolations, voir Érasme, De conscribendis..., 49, la note 3 et au texte, deux remarques proches : "Cela sera réalisé si nous atténuons avec vraisemblance le malheur même dans lequel il est tombé [...]» et surtout le développement qui suit sur la manière de montrer que le malheur «sera de courte durée » et plus précisément sur les sources des conjectures et l'état de cause conjectural (ou débat sur l'existence du fait) : «À ce point nous examinerons avec le plus grand soin toutes les conjectures par lesquelles nous rendrons cette espérance au plus haut point probable [...]. »

12. L'argument de la conjecture met en jeu les passions de l'espoir et par là de l'assurance (confidentia), à la condition expresse que ressorte la "proximité " des biens à venir (d'où l'importance de la vraisemblance). Voir Aristote, Rhétorique, 2.5, 1383a15 sq., op. cit., p. 293 : «L'assurance est l'espoir accompagné de la représentation d'une proximité de ce qui nous sera salutaire. »

13. Comprendre : par rapport à celui qu'il console. Ici commence implicitement une nouvelle section dédiée aux malheurs avérés et graves, qui obligent à un travail préparatoire important pour que le propos soit recevable et partant bénéfique. Dans le présent $\S 8$, les remarques sur l'éthos de celui qui parle s'intègrent dans cette séquence habituelle des exordes que sont les « rationes dicendi ", les raisons qui obligent à parler. Tout le développement du premier alinéa est repris de Scaliger, Poetices..., III, 122. Vossius en résume la dernière partie en la simplifiant (il passe de quatre cas à trois, éliminant la consolation philosophique). Sur le détail des propositions, voir infra, notes 20-23.

14. «Si c'est un inférieur [...] il affirmera que [...]», «Si c'est un égal, il dira que [...]»: trait stylistique constitutif de ces chapitres de Vossius sur les genres du discours, la multiplication de formules semblant "prêtes à l'emploi ». Cela ne signifie pas que le style indirect puisse être directement transformé en style direct (sur les difficultés d'analyse qu'une telle opération présente, voir B. Combette, "Discours rapporté et énonciation: trois approches différentes", Pratiques 64, déc. 1989, p.111-122) ; mais qu'en étant focalisés sur le contenu sémantique et en faisant abstraction des modulations introduites par toute énonciation, ces énoncés forgés donnent une version compacte des formulations susceptibles d'être actualisées.

15. Chapitre III, 123 dans la $1^{\mathrm{e}}$ éd. (Lyon, Antoine Vincent, 1561, p. 168-169); corrigé dans les éditions ultérieures en chap. III, 122 (par ex. : $5^{\mathrm{e}}$ éd., Heidelberg, Hieronymus Commelius, 1617, p. 387-388) ; même correction dans l'édition L. Deitz (Stuttgart-Bad, Cannstatt, 1995), p. 194-198. - Nous corrigeons le texte latin de Vossius, qui a «cap. XXIII», erreur manifeste: au §31, infra, Vossius écrit bien « cap. CXXIII». 
16. Ovide console Livie : voir la Consolation à Livie Augusta sur la mort de Drusus Néron son fils (Consolatio ad Liviam, 9 av.J.C. - 2 ap. J.-C.), parfois attribuée à Ovide. En revanche, aucune attestation pour la situation inverse (Livie console Ovide). Il faut donc comprendre: "Si Livie avait consolé Ovide, elle ne s'y serait pas prise de la même façon qu'Ovide quand il a consolé Livie ». Cette structure implicite, où une situation conditionnelle fictive est mise en parallèle avec une situation réelle attestée, sera utile pour comprendre les exemples suivants. - Le texte latin de Vossius a seulement : « aliter Naso », erreur manifeste ; nous rétablissons en " aliter Liviam Naso », conformément au texte de Scaliger.

17. Les deux exemples sont introduits par «veluti si », « comme si »: il faut donc comprendre que nous sommes du côté du conditionnel: et de fait, il n'existe aucune attestation de Pompée s'adressant à Cicéron pour le consoler. En revanche, il existe un grand nombre de lettres consolatoires de Cicéron à des proches et des lettres protocolaires de Cicéron à Pompée, dans lesquelles Cicéron exhibe un éthos de flatterie (voir en particulier Familiares, 5.7).

18. Crassus: patronyme de la famille romaine Licinia, en particulier Marcus Licinius Crassus (général et homme d'État à Rome : 115 av. J. C. - 53 av. J. C.). La famille était associée à la richesse jusque dans les stéréotypes: "Crassus le riche" (Crassus dives) était le surnom du père (P. L. Crassus) avant que d'être celui du fils (voir Cicéron, De officiis, 2.16 .57 ; De finibus, 3.75) ; et "riche comme Crassus» était passé en proverbe (voir Plutarque, Vies des hommes célèbres, Lucullus, 40). Sur la cupidité du personnage, voir le chapitre que Plutarque consacre à Crassus luimême, ibid.

19. On revient ici du côté des cas attestés. Voir, de Sénèque, la Consolation à Polybe (sur la mort de son frère) et la Consolation à ma mère Helvie (sur l'exil de son fils), auxquelles on ajoute habituellement sa Consolation à Marcia (sur la mort de son fils). Pour une reprise de ces références, voir infra, § 31 .

20. Argument à ajouter dans la liste des raisons de la captatio: le devoir de mise en garde (argument B), lequel peut se décliner de la monitio, de l'avertissement (avec recours possible aux maximes : voir ci-après le cas du sage) à l'objurgation (discours de reproches avec exhortation à s'amender). La présence du reproche dans la consolation est débattue par Érasme: celui-ci commence par l'exclure (sauf en contexte amical, voir De conscribendis..., 49: «En outre, nous prendrons garde de ne pas sembler donner des leçons plutôt qu'une consolation amicale, sauf si la personne à qui nous écrivons est un proche »); il donne ensuite in extenso un modèle de son invention intitulé "Consolation à un proche avec objurgation"; enfin il suggère une réponse cinglante à renvoyer aux donneurs de leçons (ibid.,50): "Nous répondrons ainsi à un correspondant qui nous donne des ordres: Il est facile, quand on se porte bien, de donner de bons conseils aux malades. / Si tu étais à ma place, tu penserais autrement. »

21. Le sage relève lui aussi de la catégorie éthique des supérieurs (voir supra note 20). L'argumentation en pro et contra (la disputatio) éloigne le discours du sage du grand modèle délibératif, pour le rapprocher du didactique. Voir infra note 25.

22. Autre raison de la captatio : l'affection (argument $\mathrm{C}$ ), dont le lien avec la catégorie éthique des inférieurs est à interroger. En effet, dans la société d'Ancien Régime, il existe une relation complexe entre le sentiment et le rang (social). L'attachement à des supérieurs n'est pas de nature affective, mais politique (respect, loyauté). Cependant, de la part d'un inférieur, c'est montrer un véritable attachement, qui le grandit, que d'ajouter de l'affection au respect. Mme de Sévigné nous servira ici de guide. Elle-même présente comme une exception son affection pour une princesse de sang (en l'occurrence la première d'entre elles, la Grande Mademoiselle : Lettres de l'année 1671, Paris, Gallimard, Folio Classique, 2012, p. 49, à Coulanges, le 31 décembre 1670) : «Elle est fort affligée, et m'a toujours traitée comme une personne qui sentait ses douleurs; elle ne s'est pas trompée. J'ai retrouvé dans cette occasion des sentiments qu'on ne sent guère pour des personnes d'un tel rang. » En sens inverse, en tant que maîtresse de maison, elle ressent comme une trahison affective le départ annoncé de domestiques (ibid., p. 381, à Mme de Grignan, 
le 25 décembre 1671) : «Il est vrai que les gens qui demandent leur congé serrent le cœur et font voir peu d'affection. " L'éloge d'une femme de maison sera aussi celui de son affection (ibid.) : «Elle est soigneuse, elle est affectionnée, elle a de l'honneur et de la conscience, elle est ménagère [...] »; et Picard sera autant blâmé de n'avoir point de cœur que de ne pas vouloir faner (ibid., p. 255, à Coulanges, le 22 juillet 1671) : "Je songeai que c'était la centième sottise qu'il m'avait faite, qu'il n'avait ni cœur ni affection; en un mot, la mesure était comble. »

Pour en revenir aux lettres de consolation, c'est donc ce supplément d'âme qu'en pareille occasion on attend d'un inférieur dans ses propos liminaires. Le recours aux autorités dont il est fait ensuite mention est la conséquence du défaut d'autorité attaché par nature à l'éthos de l'inférieur : parler au nom de personnes autorisées constitue à ce titre un nouvel argument visant à la captatio (argument $\mathrm{D})$.

23. Dernière raison de la captatio avancée par Scaliger : le devoir d'amitié (argument E). Quatre consolations sont ainsi distinguées chez Scaliger, par l'éthos et les caractères stylistiques induits : la consolation d'un supérieur en forme d'avertissement ; la consolation philosophique du sage, en forme de débat; la consolation de l'inférieur, autorisée par son attachement et une rhétorique des citations; enfin la consolation amicale (ou familière, pour reprendre le Ad familiares latin, littéralement «À des proches »), que Cicéron a portée au rang de genre à part entière. Si Scaliger en fait quatre types de consolation, Vossius quant à lui, dans le $1^{\mathrm{er}}$ alinéa du $\S 8$, y voit seulement divers types d'arguments éthiques à placer au niveau des rationes dicendi (ou scribendi). Autre différence, il ne retient pas la consolation philosophique du sage : sur cette exclusion, voir infra, note 25.

24. Voir Érasme, De conscribendis..., 49 : « Il faut procéder autrement, par insinuation, quand l'âme de celui qui est affligé est trop faible ou la douleur trop récente ou trop aiguë pour souffrir d'être traitée directement : et de même quand l'âme de la personne que nous consolons est trop fière pour paraître avoir besoin qu'on la console. » Reprise avec déplacement chez Vossius : il garde les distinctions éthiques affectant le destinataire, mais il ne les relie pas explicitement à un genre spécifique de consolation (la consolation par insinuation, qu'Érasme distingue de la consolation directe et franche). Mais dans les faits cela revient au même: les distinctions introduites chez Vossius par la personne du destinataire obligent à un exorde long (par insinuation) et à une adaptation des péroraisons.

25. Les deux cas semblent extrêmes, car ils s'opposent à l'écoute de la consolation : l'un, parce qu'il n'a pas besoin d'être consolé ; l'autre, parce qu'il est inconsolable. Mais c'est oublier que la consolation est d'abord une entreprise de persuasion (une contentio, un argumentaire qui vise à emporter la conviction) : ce qui signifie que si la personne n'a pas besoin d'être persuadée, alors les arguments en faveur du réconfort pourront être exposés directement, sans enjeu, sans besoin de construire un dispositif de persuasion. C'est la fameuse consolation "simple et directe " d'Érasme (De conscribendis..., 49), dont il n'y a presque rien à dire, parce qu'elle est au fond l'envers d'un discours, d'un dispositif argumentatif tendu et progressif, parce qu'elle est une simple et franche parole d'explicitation raisonnée des arguments en faveur du réconfort. Ou alors, si l'orateur opte pour un discours en forme, ce ne sera pas un discours délibératif mais un discours didactique pour philosophes et classes de philosophie, avec maximes et argumentation en pro et contra comme le suggérait Scaliger dans le passage repris par Vossius, supra $\$ 8,2^{\mathrm{e}}$ alinéa. Voir également De conscribendis..., 49: «chaque fois que nous aurons affaire à un philosophe ou en général à un homme sensé", "nous faisons voir clairement à l'aide d'arguments qu'il n'y a pas de raison de souffrir, puisque rien de fâcheux ne peut atteindre un homme sage sinon l'infamie et que nul ne peut le blesser que lui-même ».

La nécessité de persuader (le champ de la rhétorique) ne commence qu'au moment où l'on ne prêche plus la consolation à des convaincus mais à des interlocuteurs récalcitrants, soit qu'ils ne veulent pas l'entendre, soit qu'ils ne le peuvent pas. Autrement dit, les deux cas que Vossius, à la suite d'Érasme, envisage ici sont précisément les cas où il y a un besoin de persuasion, les deux 
cas qui légitiment d'abord le rituel langagier de la consolation comme dispositif fort de captation, en semblant même laisser comme secondaire (dans un des deux cas : celui de l'âme forte, voir infra § 10) le dispositif argumentatif du réconfort proprement dit.

26. De quoi l'âme forte doit-elle être consolée ? Érasme avance au préalable une interprétation, que ne reprend pas Vossius, en distinguant entre paraître et intériorité et en spéculant sur les intentions : la constance est apparente mais ce qui la motive, c'est seulement la « honte » de donner aux autres l'impression que l'on souffre (voir De conscribendis..., 49 : « ils considèrent que c'est lâche et humiliant pour un homme courageux d'être malade [...]»). Autrement dit, pour Érasme, l'homme ou la femme forte sont des malades cachés. Chez Vossius, il n'en est rien. Vossius se contente de décrire une forme de consolation qui associe au rituel du réconfort un dispositif épidictique d'admiration: de sorte que sa consolation avec éloge est tout autant susceptible d'être interprétée comme une stratégie de reconnaissance de la force d'âme montrée par la personne affligée que comme une stratégie oblique de remédiation. L'âme forte n'a peutêtre pas besoin d'être réconfortée, mais assurément d'être confortée.

27. Deux nouveaux arguments à verser dans la liste des raisons de la captatio : l'éloge de l'affligé pour sa constance (argument F) et la grandeur du mal (argument G). Amplifier le malheur est un des ressorts du pathos de la pitié, lequel est ici inversé en admiration.

28. Argument consolatoire proprement dit (en vue du réconfort) : la nécessité de souffrir et de mourir $\left(n^{\circ} 2\right)$. C'est sans doute l'argument le plus «topique ». Voir par exemple Érasme, De conscribendis..., 49 : «Rien ne soulage autant la douleur, selon Cicéron, que de se rappeler notre commune condition». Pour d'autres références sur lesquelles nous reviendrons (voir infra,

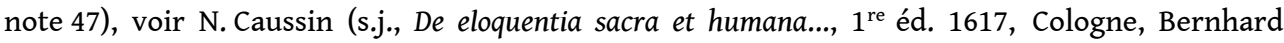
Wolter (Gualtherus), 1634, p. 611), où l'argument vient en tête de la liste des arguments communs (ibid., p. 611 : «Primum, nascendi et moriendi necessitas », " Premièrement, la nécessité de naître et de mourir »); ou ultérieurement G. J. Pelletier (s.j., Reginae Palatium Eloquentiae..., $1^{\mathrm{e}}$ éd. 1641, Lyon, J.-A. Candy, 1653, p. 818), l'argument VI noté par erreur VIII : «A communi rerum conditione ", " Argument tiré de l'universelle condition ». Pour une reprise de cet argument ici même dans le présent chapitre de Vossius, voir infra, § 27.

29. Argument consolatoire $n^{\circ} 3$ : la bonne conscience (la conscience de n'avoir pas commis de faute, de se savoir innocent du malheur que l'on subit). Voir Érasme, De conscribendis..., 49 : « tout ce qui atteint un homme sans que ce soit sa faute ne saurait être regardé comme un mal [...]. » À rapprocher d'Aristote, Éthique à Nicomaque, 1.11,1100b9 sq., trad. J. Tricot, Paris, Vrin, p. 1987, p. 73 sq. : « [L'homme vertueux] supportera les coups du sort avec la plus grande dignité et un sens en tout point parfait de la mesure, si du moins il est véritablement homme de bien et d'une carrure sans reproche [N.d.E. : vers de Simonide]. » Argument récurrent du stoïcisme antique. Voir à titre d'exemple Sénèque, Lettre 74 à Lucilius (dans Les Stoïciens, trad. E. Bréhier, éd. sous la dir. de P.-M. Schul, Paris, Gallimard, « La Pléiade », 1962, p. 793) : « Quiconque s'est beaucoup livré au jeu de la fortune s'est préparé de très grands, d'inextricables sujets de trouble : la seule voie pour se mettre en sûreté consiste à mépriser les choses extérieures et à se contenter de ce qui est honnête. Quiconque pense qu'une chose est meilleure que la vertu, ou qu'un autre bien existe, présente le pli de sa toge aux hasard de la fortune [...]. » Sur l'imprégnation stoïcienne du présent $\S 10$ et la coloration héroïque de cette attitude de fermeté, voir à l'incipit l'usage du terme constantia dans la formule : «bien qu'il s'agisse d'un malheur qui pourrait entamer la constance de n'importe qui d'autre »

30. Le verbe gratulare est à mettre en relation avec l'oratio gratulatoria, le discours de congratulation que Vossius traite dans la série des discours démonstratifs (Rhetorices contractae..., II, 19). La gratulatio peut à la fois être la visée générale d'un discours entier ou, comme ici, une séquence intégrée concourant à la visée persuasive d'un autre type de discours, en l'occurrence la consolation. Il en sera de même, $\S 24$, pour l'adhortatio conclusive, séquence exhortative à rapprocher du discours d'exhortation. 
31. Vossius résume ici le De conscribendis. Il reprend à Érasme les quatre étapes du plan : 1 . amplifier le malheur de son interlocuteur ; 2. faire l'éloge de sa grandeur d'âme ; 3 . énoncer les raisons du réconfort sur le mode prétéritif (la nécessité de mourir, la bonne conscience); 4. présenter ses félicitations. Voir Érasme, De conscribendis..., 49 : «nous dirons que nous ne lui écrivons pas pour le consoler, car nous avons pu constater de multiples manières sa sagesse remarquable et son inébranlable grandeur d'âme, qui est plus forte que tous les orages de la fortune; et que même si nous admettons que son malheur est tel qu'il aurait facilement mis à terre n'importe qui d'autre, cependant nous ne doutons pas qu'un homme élevé depuis l'enfance dans les préceptes de la vraie philosophie, instruit par une longue expérience, et muni, contre ces épreuves, d'une force de caractère invincible supportera avec courage ce qui ne peut être évité, puisque c'est là la loi commune qui s'impose aux mortels. Nous dirons que nous voulons le féliciter pour sa fermeté, plutôt que remédier à sa douleur. " Il est à noter qu'il ne s'agit pas là de quatre parties d'égal volume: dans le modèle de lettre que forge Érasme pour illustrer le cas (la Consolation à Canidius sur son exil, non traduite dans l'ATELIER), sur les huit pages de l'édition de 1522 (op. cit., p. 242-250), les points 1. et 2. occupent les premières phrases de la lettre (soit une page : exorde insinuatif long), les félicitations la « conclusio» (un quart de page), et le point 3. (les raisons du réconfort) presque sept pages. C'est donc là la partie centrale : il est clair qu'Érasme n'en reste pas à deux arguments consolatoires mais procède par accumulation. D'où la possibilité d'interpréter les deux arguments ici mentionnés par Vossius non pas comme une liste exhaustive, mais comme deux cas exemplaires des « préceptes salutaires de la philosophie ».

32. De quelle maladie souffre l'âme faible? De nouveau, Vossius ne reprend pas l'interprétation d'Érasme et propose directement un plan (au double sens de plan d'action et de canevas). Érasme en effet assimile la faiblesse de l'âme (la dépression) à une démence. Voir De conscribendis..., 49 : " ceux que la douleur possède tout entiers ne doivent pas être traités différemment de ceux qui, du fait d'un manque de jugement dû à la maladie, pensent qu'ils ont des cornes [...] ». Et la cure psychologique qu'il met alors en place s'appuie sur les notions de transfert (ibid.: "nous remédierons en transférant sur nous-mêmes [in nos transferamus] les sentiments de la personne que nous voulons consoler [...]») et d'imitation (voir ibid., le couple dissimulatio / simulare : "par dissimulation [...] ceux qui s'efforcent de leur apporter des remèdes simulent parfois qu'ils sont eux-mêmes victimes du même mal »).

33. Le plan que propose Vossius correspond à celui d'Érasme: d'abord une partie dédiée à l'amplification du malheur et au témoignage de sa propre souffrance, c'est-à-dire à la captation de l'écoute (chez Vossius : §11-14); puis une partie dédiée aux raisons du réconfort (\$15-23); enfin, partie non annoncée ici, l'exhortation à se ressaisir (\$ 24). Dans les lettres modèles forgées par Érasme, les volumes consacrés à chacune des parties permettent là aussi de les requalifier : la première partie est de nouveau la matière d'un exorde insinuatif ; la dernière partie occupe la péroraison; et la partie centrale est l'argumentation consolatoire proprement dite. La consolation que compose Érasme pour illustrer le cas (la Consolation à Antoine Sucquet sur la mort de son fils, non traduite dans l'ATELIER) en témoigne : sur les 15,33 pages de l'édition de 1522 (op. cit., p. 250-266), un tiers de page est dévolu au mouvement compassionnel (incipit de l'exorde); l'exhortation finale prend une page $3 / 4$, sous la forme particulièrement habile d'une prosopopée (le fils mort reprochant au père sa douleur et l'appelant à se ressaisir : «quaeso per vestram in me pietam ", «je vous en conjure par la piété que vous avez pour moi »); et les raisons du réconfort s'étendent en continu sur 12,5 pages avant d'être récapitulées dans un «Épilogue » placé après l'exhortation ( $3 / 4$ de page).

34. Vossius recense quatre micro-séquences pour composer l'exorde d'une consolation à un affligé, qui fournissent de nouvelles raisons visant à la captatio : 1 . témoignage que nous-mêmes souffrons ( $\S 11$ : notre propre douleur, argument $\mathrm{H}) ; 2$. amplification de notre douleur $(\S 12) ; 3$. amplification du malheur ( $\$ 13$ : la grandeur du mal, argument $\mathrm{G}$ déjà rencontré supra $\S 10$ dans la consolation aux âmes fortes); 4. témoignage que tous les honnêtes gens souffrent en pareil cas 
(§14). La grandeur du malheur met en jeu le pathos de la pitié, le témoignage de notre propre douleur celui de l'amor (c'est une preuve d'affection que de souffrir avec ses amis).

35. Sur cette mention de l'éloge, voir le chapitre de Ménandre le Rhéteur sur la consolation (Vossius cite en fin de son propre chapitre cette référence : voir infra §31). Ménandre en effet, tout en ne considérant que la consolation adressée à l'âme éplorée (« Le discours de consolation pleure, lui aussi, celui qui n'est plus [...]» : trad. H. Caffiaux, op. cit., p. 277), y insère des séquences d'éloge. Toutefois, il ne s'agit pas chez lui de l'éloge de celui qui souffre mais du défunt. Pour en revenir à Vossius, on peut noter que dans les deux cas successivement envisagés de l'âme forte et de l'âme éplorée, il préconise à des degrés divers l'éloge de celui qui reçoit la consolation.

36. Voir Érasme, De conscribendis..., 49 : « et nous dirons, non sans quelque éloge, que nombre de gens de bien souffrent de l'injustice qui l'accable.» Dans le texte de Vossius, on remarquera la structure cumulative de l'ensemble: nous commençons par témoigner de notre propre douleur (d'où amor); comme preuve de sa réalité, nous l'amplifions par les effets (argument ab effectis); comme preuve de son ampleur, nous amplifierons le malheur qui en est à l'origine (argument $a$ causa; d'où pitié) ; comme preuve que le malheur est une injustice, nous nous appuyons sur le témoignage des gens de bien (argument a consequentia). L'ensemble rappelle ce mode du raisonnement que la logique et la rhétorique nomment sorite. Le sorite est un raisonnement composé long, opérant par propositions enchaînées (voir A. Arnaud et P. Nicole, La Logique ou l'art de penser, $1^{\mathrm{e}}$ éd. 1660, $5^{\mathrm{e}}$ éd. 1680, Paris, Flammarion, 1970, coll. «Champs », 1991, Part. III, chap. 1, p. 235).

37. Vossius décrit ici un processus d'imitation, centré sur « aequo [...] animo», «d'une âme égale ». Voir Scaliger, Poetices..., III, 122 : « Nous persuadons mieux l'auditeur d'acquiescer, s'il voit que nous supportons ce qui arrive d'une âme égale, à condition qu'il comprenne bien que nous aussi partageons sa douleur, nous qui lui donnons tant de poids. » À rapprocher du processus de purgation par transfert et simulation qu'Érasme met en place (voir supra, note 32).

38. Voir Scaliger, Poetices..., III, 122 : «Celui qui aime ne peut que détester les propos de ceux qui paraissent accorder peu de valeur à la perte de la personne ou de la chose aimée. » C'est l'un des lieux de la colère selon Aristote, Rhétorique, 2.2, 1378b15-20, op. cit., p. 269 : «le malade, par exemple, contre ceux qui déprécient sa maladie, [...] l'amoureux, contre ceux qui dédaignent son amour et ainsi de suite.» Voir également Érasme, De conscribendis..., 49 : « Ils [ceux qui sont atteints de démence] détestent ceux qui les contredisent [...]».

39. Ici finit l'exorde et commence la partie centrale et fondamentale de la consolation, en tant qu'elle est un dispositif argumentatif rassemblant les raisons d'adoucir la douleur et visant au réconfort (\$15-23). C'est en raison de ce but, réconforter (littéralement, rendre plus fort) que Keckermann nomme les consolations des « discours de réconfort». Voir Kerckermann, Systema rhetoricae, op. cit., p. 667 et p. 675 (« [oratio] firmatoria et consolatoria », « discours de réconfort et de consolation »). On notera que Vossius déploie bien plus longuement la consolation pour les âmes éplorées (et en particulier la partie « réconfort ») que la consolation pour les âmes fortes, réglée en un paragraphe (voir supra, § 10).

40. Ici et ensuite (\$ 22, 24 et 31), la «patience » est une vertu que Vossius dirait virile, c'est le courage de supporter, d'endurer, sur le verbe patior; chez Cicéron (De inventione, 2.163), la patientia est une des quatre parties du courage ou fortitudo, soit «grandeur d'âme, confiance en soi, patientia, perseverantia» (voir ici, au $\S 31$, le doublon patientia et fortitudo). - Vossius a distingué (supra §5) deux types de maux : remédiables ou irrémédiables. Nous sommes bien ici dans cette seconde catégorie. Dans le cas des malheurs d'importance, deux stratégies sont ici convoquées : agir sur la douleur elle-même (c'est, ici-même, l'argument $n^{\circ} 4$ : la patience, voir infra note suivante) et atténuer le malheur lui-même (voir infra §16). Érasme distingue deux manières d'atténuer (De conscribendis..., 49) : «Une fois que cela aura été fait, nous appliquerons le remède; à cet endroit nous rassemblerons avec soin tous les arguments qui sont susceptibles d'apaiser la douleur. Cela sera réalisé si nous atténuons avec vraisemblance le malheur même 
dans lequel il est tombé et si nous minimisons, autant qu'il est possible, le bonheur d'où il est tombé. » Vossius ne conserve ici (§ 15-16) que l'atténuation du malheur lui-même.

41. Note de Vossius : «Lib. I. Od. 24.» Horace, Odes, 1.24.19-20, trad. Ch.-M. Leconte de Lisle, Paris, A. Lemerre, 1911. On remarquera que l'argument de la patience semble venir hors système, sans faire série avec d'autres: en vérité, Vossius le développera ultérieurement (\$22) en l'intégrant dans le système qu'il met en place juste infra $§ 17$. Pourquoi l'avoir tiré de cette liste et l'avoir répété en quelque sorte en exergue à l'orée du développement? Peut-être est-ce un souvenir du modus operandi de Ménandre le rhéteur (2.9, «Du discours de la consolation », op. cit), qui propose de placer en tête des consolations quelques vers d'Euripide.

L'important - pour l'item ouvrant la liste des arguments consolatoires proprement dits - ne serait donc pas le contenu argumentatif mais la forme, non pas, par exemple, la préconisation spécifique de la patience, mais l'indexation de toute la démarche sous la tutelle de la poésie : car la parole consolatoire n'est pas qu'une parole particulière, mais aussi une parole communautaire et protocolaire ; et son efficacité tient autant à sa ritualité (à son ornatus) qu'à son decorum, qu'à l'adaptation de son contenu sémantique.

L'hypothèse semble devoir se vérifier avec un prédécesseur immédiat de Vossius, qu'il cite luimême à d'autres occasions, le jésuite Caussin (De eloquentia sacra..., op. cit., p. 611) : en tête des arguments consolatoires "communs" (communs à toutes les consolations), Caussin insère lui aussi une petite dizaine de vers (en l'occurrence, de Stace, sur la nécessité de mourir).

42. Trois arguments sont ici énoncés ( $n^{\circ} 1$ : la conjecture, déjà rencontrée supra $\S 7 ; n^{\circ} 5$ : les suites heureuses; $n^{\circ} 6$ : les malheurs plus grands), sans être formalisés selon la grille des lieux communs. Il est cependant possible de la restituer : nous avons d'abord un argument tiré de la circonstance du temps (le futur) ; puis un argument tiré des conséquences (possibilité de suites heureuses, d'un bien, argument développé dans le De conscribendis..., 49: «On le fera en mentionnant précisément, avec quelque amplification, tous les avantages qui en résulteront. Car il n'y a pas de mal si grave qui puisse survenir dans la destinée humaine qu'on ne puisse en récolter un avantage ») ; enfin un argument tiré d'une comparaison a majori. Les arguments de la conjecture et des suites heureuses mettent en jeu le pathos de l'espoir ; l'argument des malheurs plus grands joue quant à lui sur le pathos de la honte (pudor).

43. La locution adverbiale marque une opposition avec le $\S$ précédent: nous pouvons certes atténuer le malheur en tirant argument des lieux communs (c'est-à-dire des sources d'argumentation communes aux trois grands genres de discours) ; mais « avant tout " ( imprimis $»)$, nous préférerons les arguments tirés des sources spécifiques du seul genre délibératif, telles qu'énumérées ci-après.

44. Ce sont ici quelques-uns des lieux spécifiques du genre délibératif, que Vossius a recensés dès le premier livre de ses Rhetorices contractae... (I, 5 « De genero deliberativo", § 5-14). Nous en rappelons la liste entière : 1 . argument tiré du légitime; 2 . de l'équitable (et du pieux); 3 . de l'utile (et du nécessaire ainsi que de l'agréable) ; 4 . du possible (et du facile ainsi que du difficile); 5. du glorieux (et du célèbre ) ; 6 . de l'issue (ab eventu). Vossius active dans le présent chapitre :

- le lieu de l'équitable, décliné ici sous la catégorie de l'honorable §18, avec les arguments de la Providence et de la bonne conscience ;

- le lieu de l'utile, décliné ici sous les catégories de : l'utile (\$19) avec les arguments de la réputation accrue par le malheur et de la bonification morale par l'épreuve; le nécessaire (\$ 20) avec l'argument de l'irritation du mal par les pleurs; l'agréable (\$21) avec l'argument du souvenir futur du malheur présent ;

- et le lieu du possible (\$22-23), avec, à nouveau, l'argument de la patience, associé à des exemples de constance.

45. Arguments de la Providence, de la volonté de Dieu $\left(n^{\circ} 7\right)$ et de la bonne conscience $\left(n^{\circ} 3\right):$ si nous n'avons rien à nous reprocher (si nous avons pour nous la voix de la conscience) ou si c'est 
la volonté de Dieu (la Providence), alors le malheur qui nous atteint n'atteint pas notre vertu, ce qui est une satisfaction. Sur l'argument de la bonne conscience, voir supra, § 10 et note 29.

46. Deux arguments : la réputation accrue par le malheur $\left(n^{\circ} 8\right)$ et la bonification morale par l'épreuve $\left(\mathrm{n}^{\circ} 9\right)$. Voir Érasme, De conscribendis..., 49 : « un tel malheur bénéficiera à sa réputation ou est un jalon posé dans la direction d'un très grand avantage. La plus grande vertu et la plus grande félicité commencent le plus souvent avec les débuts les plus difficiles [...]. »

Rappelons que le De conscribendis..., 49 série sans les formaliser les arguments suivants (pour la partie « Réconfort » proprement dite) : les suites heureuses (« les avantages qui [...] résulteront », argument $n^{\circ} 5$ chez Vossius), la bonne conscience ( tout ce qui atteint un homme sans que ce soit de sa faute", argument $n^{\circ} 3$ chez Vossius), la réputation accrue par le malheur (« un tel malheur bénéficiera à sa réputation ", argument $n^{\circ} 8 \mathrm{chez}$ Vossius), la bonification morale par l'épreuve ( « la tempête envoyée [...] est [...] salutaire pour l'âme », argument $n^{\circ} 9$ chez Vossius), la nécessité de souffrir et de mourir ("se rappeler notre commune condition », argument $n^{\circ} 2 \mathrm{chez}$ Vossius) et l'argument par les exemples de constance («ce malheur, il le partage [...] avec une foule d'hommes réputés", argument $n^{\circ} 12$ chez Vossius : voir infra § 23). Érasme préconise ensuite de conclure par l'exhortation («Enfin nous en viendrons à l'exhortation ») : même règle chez Vossius, infra $\$ 24$.

47. Voir Sénèque, De la Providence (dans Les Stoïciens, op. cit., p. 762) : «Entre bien des paroles magnifiques de notre ami Démétrius [N.d.E. : Philosophe cynique], en voici une que j'ai entendue récemment et qui résonne encore et retentit à mes oreilles: Nul être, dit-il, ne me paraît plus malheureux que celui qui n'a pas été touché par l'adversité. »

Le jésuite Pelletier se souviendra de l'exemple et plus généralement des deux sources de la consolation que Vossius répertorie ici, l'honorable et l'utile (voir Reginae Palatium..., op.cit., p. 817). Plus exactement, en respectant leur ordre et leur place inaugurale dans la suite des lieux spécifiques du délibératif consolatoire, Pelletier les détaillera en quatre items. L'honorable donnera lieu aux deux premières entrées, l'une pour ce que nous avons repéré comme l'argument de la Providence (ibid.: «I. Divina Providentia », «La Providence divine ») ; l'autre, sous l'intitulé générique "II. Ab honesto», "Argument tiré de l'honorable», développera l'argument de la bonne conscience, avec une reprise quasi littérale de Vossius (ibid. : Nimirum hoc virtus et honestas exigit, ut dolorem recte factorum conscientia sublevemus ", " assurément la vertu et l'honnêteté l'exigent : ainsi nous allégerons la douleur de son âme par la conscience d'avoir agi de façon juste »). Quant à l'item de Vossius «Ab utili», Pelletier le divisera de nouveau en deux, en spécifiant d'abord l'argument de la réputation accrue par le malheur (ibid. : «III. A laude et gloria », «Argument tiré de l'éloge et de la gloire »); et en reprenant sous l'item «IV. Ab utili» ("Argument tiré de l'utile») la seconde proposition de Vossius sur la bonification par l'épreuve (ibid. : "ad prudentiam et fortitudinem », " [les malheurs forment] à la prudence et au courage »), avec le même exemple d'Alexandre (ibid. : " hoc uno censetur infelix Alexander quod semper fuerit felix ", « On juge que le malheur d'Alexandre est d'avoir toujours été heureux »).

Sans continuer à suivre l'ordre de Vossius, Pelletier décline ensuite six autres items dont certains recoupent ceux de Vossius : «A feliciori mortuorum statu ( ( Argument tiré de l'état bienheureux des morts »); «A communi rerum conditione » («Argument tiré de l'universelle condition »); «Ab ejusdem fortunae societate» («Argument tiré de ce que cette même infortune est partagée par d'autres »); «Ab opinione sapientum» («Argument tiré de l'opinion des sages »); «Ab illustribus eorum exemplis» («Argument tiré des exemples illustres [de constance]»); «A mortis bono ( Argument tiré du bienfait de la mort »). Et il centrera enfin la péroraison sur l'exhortation, comme avant lui Érasme et Vossius.

Sur les liens entre Vossius et les jésuites, rajoutons que Vossius lui-même fait référence à Nicolas Caussin; mais ce dernier propose une autre liste des sources du consolatoire délibératif (De eloquentia sacra..., XII, XI, op. cit., p. 611-612) : 1. la nécessité de mourir ; 2. la vie est un supplice ; 3. les malheurs plus grands ; 4 . la fragilité des biens terrestres ; 5 . la mort est une délivrance ; 6 . la 
félicité de la vie dans l'au-delà ; 7. gémir est honteux ; 8. les exemples de constance.

Notons enfin que Caussin, Vossius et Pelletier classent donc tous les trois les arguments du réconfort en fonction des lieux du délibératif. Pelletier s'en tient là, alors que Caussin et Vossius ajoutent d'autres méthodes de consolation. Voir infra note 56.

48. Argument $n^{\circ} 10$ de l'irritation du mal par les pleurs. Dans ses Institutiones, Vossius le mentionnait par deux fois. Voir Institutiones, op. cit., III, 4, p. 394 : «Denique dicemus stulti esse animum angere frustra: moerorem autem ejusmodi esse, ut non corrigat rem; sed malum, siquod est, augeat. » Et ibid., p. 396 : «Propterea non debere nos angi, ubi nihil proficimus. Quin moerore malum aggravari. " Argument souvent étayé par une référence à l'anecdote d'Arsinoë (Plutarque, Consolation à Apollonius sur la mort de son fils, 112a-b) : pleurer, c'est entretenir le culte du dieu Deuil, lequel vous reste alors attaché ; cesser les pleurs, c'est le faire fuir. Pour une paraphrase de la fable, voir Caussin, De eloquentia sacra..., op. cit., p. 612.

49. Aulu-Gelle, Nuits attiques, 17.14, "Choix des meilleures pensées des comédies de Publius [Mimus]», trad. M. Charpentier et M. Blanchet, Euvres complètes d'Aulu-Gelle, t. II, Paris, Garnier, 1920. Il s'agit de maximes versifiées.

50. Vossius compacte deux arguments : à la façon d'Érasme (De conscribendis..., 49 : « doux est le souvenir des maux passés, quand ils sont associés à une réputation honorable", "malorum recordationem quae cum honesta fama coniuncta sunt »), il associe l'argument de la bonne conscience $\left(n^{\circ} 3\right)$ avec un nouvel argument, le souvenir futur du malheur présent ( $\left.n^{\circ} 11\right)$. Mais alors qu'Érasme le développe surtout en liaison avec la vertu (voir supra note 29), Vossius met l'accent sur la douceur liée à la remémoration, dans les pas de la Rhétorique d'Aristote. Voir Aristote, Rhétorique, 1.11, 1370 b1 sq., op. cit., p. 215 : « Les choses dont le souvenir est plaisant, ce sont non seulement celles qui étaient plaisantes sur le moment, quand elles étaient présentes, mais pour certaines, des choses qui n'étaient pas plaisantes, si ce qui a suivi plus tard a été beau et bon, ce qui a fait dire : Il est plaisant, une fois sauvé, de se rappeler les dangers qu'on a courus [N.d.E. : Euripide, Andromède, fr. 131 Nauck] et: Car après coup, lorsqu'il se souvient, il prend plaisir même à ses souffrances, l'homme qui a beaucoup subi et beaucoup accompli [N.d.E.: d'après Homère, Odyssée, v. 400-401]."

51. Reprise de l'argument $n^{\circ} 4$, placé en exergue du système au $\S 15$ (où il est orné d'une citation d'Horace). Voir supra notes 40 et 41 . Ici même, dans le paragraphe suivant (\$23), Vossius associe la patience aux "exemples éclatants de ceux qui ont supporté l'adversité avec constance». Patience et constance renvoient évidemment au stoïcisme. Érasme explicite la référence dans le De conscribendis..., 49 : "C'est ici que les arguments des philosophes et particulièrement des stoïciens seront d'une très grande aide. » Vossius quant à lui insère une référence aux trois consolations de Sénèque à la fin de son chapitre (voir infra, § 31). Dans les Institutiones (op. cit., p. 394), il renvoyait également à Épictète («Vide praeter Senecam et Epitectum [...] », « Voir surtout Sénèque et Épictète $[. .] »$.$) .$

52. La syntaxe laisse à penser que les exemples viennent étayer l'argument précédent. Mais la numérotation des paragraphes nous indique autre chose : depuis le $\S 17$, Vossius introduit un alinéa numéroté chaque fois qu'il change de catégorie d'arguments. Or c'est ce qu'il fait ici. Et de fait, même si la liaison thématique est évidente avec la patience, les exemples de constance constituent bien une ultime ressource argumentative à part entière (argument $n^{\circ} 12$ ). Voir Érasme, De conscribendis..., 49, dernier argument recensé : «À ce point, nous multiplierons les exemples d'hommes célèbres [...]. » Voir également Caussin, De eloquentia sacra..., op. cit., p. 611, huitième et dernier argument commun ("Octavo [...] subjiciuntur exempla eorum [...]», « Huitièmement suivront les exemples de ceux qui [...]»). Voir enfin Pelletier (Reginae Palatium..., op.cit., p. 819), l'avant-dernier argument: «Argument tiré des exemples illustres parmi les sages ». C'est là un argument qui met en jeu le pathos de l'émulation. 
53. Pour un semblable accent mis sur l'efficacité des exemples pris parmi les nôtres et non les étrangers («domestica»), voir Caussin, De eloquentia sacra..., op. cit., p. 611 : « [...] exempla [...] quae quo magis domestica sunt, et nota, plus habent impressionis [...]. »

54. L'exhortation constitue pour Vossius le dernier mouvement des consolations à l'usage des âmes faibles, là où la consolation pour les âmes fortes se concluait sur une séquence congratulatoire (voir supra $\S 10$ ). Retrouvant une nouvelle fois les formules de Vossius (par exemple « pristinam... virtutem »), Pelletier en fera un « Epilogus» en forme d'avertissement (voir Reginae Palatium..., p. 820: «Epilogus. Habet exhortationes quibus moerentem monemus [...]»; «Épilogue. Avec exhortations, par lesquelles nous adressons une mise en garde à ceux qui sont affligés ») ; et Érasme fait suivre ce dernier mouvement exhortatif d'une brève séquence propre à l'épistolaire (les offres de service, "En conclusion nous offrirons toute notre assistance », etc. : voir De conscribendis..., 49).

55. Vossius récapitule ici, en introduisant des variations, deux arguments consolatoires: l'argument $n^{\circ} 10$ (l'inutilité des pleurs), l'argument $n^{\circ} 8$ (éviter la honte de souiller sa gloire). Il en ajoute un nouveau, "revenir à la vertu des anciens", qui participe d'une idéologie aristocratique et dont on retrouvera une variante infra § 28 sous la forme d'un appel à renouer avec une fermeté virile (argument $\mathrm{n}^{\circ} 13$ ), incluant la "patience» au sens de la virile patientia. L'ensemble vient conclure une analyse qui avait commencé de façon étroite au § 17 («Nous tirerons ici [...] nos arguments des lieux de l'honorable, de l'utile, du nécessaire, de l'agréable, du possible ») et de façon plus large au § 11 ( $\mathrm{Si}$ au contraire son âme est faible [...], le discours sera en deux parties »). La nouvelle méthode introduite immédiatement après sera également à entendre par rapport à ce seul cas.

56. Comme pour d'autres genres du discours (par exemple pour le discours de mariage en II, 18), Vossius propose deux plans en parallèle pour la consolation à usage des âmes faibles et éplorées. La première méthode (\$ 11-24) proposait une partie consacrée à l'insinuation en vue de la captatio et une partie consacrée à l'accumulation d'arguments pour réconforter, tirés des sources du délibératif. Dans quel sens peut aller la différence que Vossius revendique pour sa seconde méthode ? On peut penser à la division de Caussin en arguments « communs » (à l'ensemble des consolations) et en arguments "privés» («privata») ou "singuliers» (« singulares»), tirés des circonstances particulières de la vie et de la famille du défunt ( ab adjunctis defunctorum »). Rien de tel chez Vossius. Même si les arguments des §27-31 sont focalisés sur la personne du destinataire, ils ne renvoient pas à des circonstances privées mais projettent au contraire un moule éthique universel. On peut alors rapprocher cette nouvelle division de l'une des distinctions opérées par B. Keckermann dans son Systema rhetoricae (op. cit., p. 706) entre deux catégories d'arguments : "Argumenta confirmantia, erigentia, sive consolantia; vel generaliora sunt; vel specialiora ", "Les arguments pour réconforter, renforcer, ou encore consoler, sont ou bien généraux ou bien spéciaux ». Il identifie les arguments généraux aux arguments tirés des sources du délibératif ou même des lieux communs (à la façon des Rhetorices contractae... : ici même, supra, $\S 15-23)$. Et il définit ainsi les arguments spéciaux (ibid., p.607): "Specialia vero argumenta ducuntur a pio, atque adeo a religione Christiana [...] ", "Les arguments spéciaux sont tirés de la piété, et plus précisément de la religion chrétienne [...]». Par la coloration chrétienne que Vossius donne lui aussi à sa seconde voie et par les exemples qu'il prend (voir infra § 27, 28, 29, 30 et 31 ), cette nouvelle version de la consolation pour les âmes éplorées s'avère plus étroitement une consolation pour les âmes endeuillées.

57. Viam inire (" emprunter le chemin ») : la métaphore du parcours est absolument cardinale en rhétorique, en liaison avec une conception dynamique du discours comme dispositif progressif de persuasion. C'est le thème connu du ductus, de la conduite du destinataire, de l'exorde à l'argumentaire et à la péroraison; et à l'intérieur de l'argumentaire, d'un argument à l'autre. Dans le premier canevas, s'il apparaissait avec netteté que l'exorde était stratégique (avec son réglage des raisons d'écrire et surtout ses arguments en vue de la captatio), l'effet cumulatif ne 
semblait pas évident au niveau de la succession des arguments consolatoires tirés de l'honorable, de l'utile et du possible ; et l'on pouvait hésiter à savoir si c'étaient des items au choix ou bien alors corrélés. Seul l'argument des exemples de constance $\left(n^{\circ} 12\right)$ était assigné à une place, venant couronner l'ensemble avant la péroraison. Il en ira différemment dans le nouveau « chemin » qui s'ouvre ici : d'abord il s'agit bien d'un canevas entier, de l'exorde (infra § 25) à l'argumentaire (infra § 26-31: on notera l'ellipse de préconisations concernant la péroraison); mais à l'intérieur des arguments, le défilé s'ordonnera selon une logique cumulative progressant pas à pas et récapitulée infra $\S 31$.

58. On reconnaît la catégorie des "raisons qui obligent à parler (ou à écrire) ", où se règle en particulier l'éthos de celui qui console : nous sommes donc dans l'exorde. Vossius récupère ici en les résumant quelques-uns des arguments visant à la captatio benevolentiae qu'il avait repris de Scaliger (voir supra § 8) et d'Érasme (voir supra § 11-14) : le devoir d'amitié (argument D), la pitié (associée à la reconnaissance de la grandeur du mal : argument $\mathrm{G}$ ), le devoir d' "encouragement » que l'on peut rapprocher du devoir de mise en garde (argument B). Pour le lien entre amitié et mise en garde, voir Érasme, De conscribendis..., 49 et supra note 20. Vossius ajoute ici à la liste l'argument de la parenté (argument I).

59. Suite de la captatio benevolentiae : l'argument $\mathrm{G}$ (la grandeur du mal : d'où le pathos de la pitié) et l'argument $\mathrm{H}$ (notre propre douleur, d'où le pathos de l'amor, de l'affection). À mettre en parallèle avec l'exorde réservé aux âmes en grande souffrance (voir supra, §11-14): le présent $\S 25$ résume les $§ 12-13$.

60. Suite de l'exorde, toujours très proche de l'exorde proposé dans la première méthode (supra $\S 14,2^{\mathrm{e}}$ alinéa). Le présent $\S 26$ reprend l'amplification du malheur (voir $\S 13$ ) et la possibilité de ne pas en être brisé (stratégie de l'imitation : voir supra § 14, deuxième alinéa).

61. Ici commence l'argumentaire du réconfort: il diffère radicalement de l'argumentaire de la première méthode. Dans la première méthode, le raisonnement portait d'abord sur le malheur, sa gravité (supra § 15-16) et sur ce qu'il est honorable, utile, possible, d'en faire (supra § 17 et sq.). Dans la seconde méthode, le raisonnement porte sur la personne du destinataire (ici même, § 27). Il s'agit là d'une nouveauté : Vossius innove en proposant de raisonner sur celui qui souffre, sur son éthos, et non pas sur la nature de la perte ou ses conséquences. Il met en place les mécanismes d'une remédiation éthique, qui passe par la proposition d'un modèle de comportement progressivement affiné (en cinq étapes). On avait vu qu'à la suite d'Érasme, la démarche consolatoire était associée à une pratique du transfert et de la simulation de la part de celui qui console (voir supra, note 32). Vossius ajoute ici un nouveau volet, celui de la conversion (au sens de renouer plus étroitement avec Dieu, de raviver sa spiritualité). Pour celui qui souffre en effet, rectifier progressivement son éthique constitue une véritable ascèse, qui va de la morale païenne (infra § 27-29) jusqu'aux plus hauts degrés de la morale chrétienne (infra § 29-31).

62. Reprise de l'argument $n^{\circ} 2$ (la nécessité de souffrir et de mourir: voir supra note 28). Argument commun à la sagesse antique et à la sagesse chrétienne. Plus précisément, Vossius semble ici paraphraser une lettre familière de Cicéron. Voir Familiares, 5.16.2 (trad. M. Defresne, sous la dir. de M. Nisard, Paris, Dubochet, t. V, 1843) : «Nous ne devons pas oublier que nous sommes hommes; que la loi de notre naissance est de vivre en butte à toutes les épreuves; que nous n'avons pas le droit de refuser la condition sous laquelle nous naissons et vivons [...]. » Avec l'adverbe « constanter » (« avec constance »), Vossius associe clairement l'argument à l'injonction stoïcienne : et l'argumentaire qu'il propose commence ici sous la forme de la mise en garde (" monebimus »), dont on se rappelle qu'elle est caractéristique du philosophe (voir supra $\S$ 8) et plus généralement de ceux qui donnent des leçons.

63. Vossius considère les femmes et ceux qui aiment, " amants " dans le français du XVII ${ }^{\mathrm{e}}$ siècle (qu'ils soient maris ou amis) comme emblématiques du luctus, ce deuil nécessairement accompagné de ses signes extérieurs, à commencer par les pleurs « lugubres » (sur lugere, comme luctus) : voir également le chapitre sur l'oraison funèbre (Rhetorices contractae..., II, 16, § 9 et $\S 16$ ). 
64. Voir Caussin, De eloquentia sacra..., op. cit., p. 611 : «Ad haec inferetur superbae esse impotentisque arrogantiae, ut quis in hac naturae necessitate [...] se unum ac suos seponi velit. " " De là on en déduit qu'il y a une arrogance orgueilleuse et impuissante à vouloir s'exempter, soi-même et les siens, [...] de cette nécessité naturelle. »

65. Litt. « tout ce qui est de cette sorte ». La séquence de poésie que Ménandre place à l'incipit des arguments visant au réconfort (un fragment d'Euripide: Cresphontès, frag. 542, Paris, Garnier, 1954) déploie le même argument. Voir Ménandre, op. cit., p. 277 : «Vous commencerez à peu près de cette manière : J'aurais lieu de m'étonner, ô parents ici présents, s'il ne vous était pas venu à l'esprit quelque souvenir de ce que dit l'excellent poète Euripide, qui fut véritablement le nourrisson chéri des Muses : Il faut, dit-il, quand un homme vient au monde, pleurer en songeant au devant de quels maux il s'avance [...]. »

66. Note de Vossius (au mot «Papinius" qui précède la longue citation) : «Lib. 2. Sylvar. in Epicedio Glauciae. » Stace, Silves, 2.1.209-219, "Chant funèbre pour Glaucias Melior ", trad. H. Clouard, Paris, Classiques Garnier, 1935. Vossius reprend in extenso l'exemple que Caussin a retenu pour étayer l'argument. Il est à noter qu'il ne s'agit pas d'une consolation, mais, comme le rappelle Caussin (De eloquentia sacra..., p. 611) d'un " épicède », autrement dit d'une des variantes de l'oraison funèbre. L'oraison funèbre prononcée au moment du décès peut partager avec la consolation une séquence conclusive de réconfort et d'exhortation à se ressaisir (voir Vossius, Rhetorices contractae..., II, 16, § 19) ; elle en diffère par l'ampleur qu'elle accorde aux séquences d'éloge, d'où le fait que l'oraison funèbre relève du genre démonstratif. Sur le choix, par Caussin et Vossius, de cette pièce de poésie dont le genre pose problème, pour illustrer un argument que l'on pouvait trouver dans bien d'autres écrits, voir supra note 41.

67. Vir: terme polysémique, mais qui est ici incontestablement «genré ». Ce n'est pas tant «l'homme de courage» cher à la traduction du XIX ${ }^{\mathrm{e}}$ siècle dans l'extrait de Cicéron juste ciaprès, qu' « un homme viril », ou comme il est dit à la phrase suivante, « un mâle » (mas, maris, m.). Dans le contexte de survivance des valeurs aristocratiques dont Paul Bénichou a montré l'emprise tout au long du XVII ${ }^{\mathrm{e}}$ siècle (Morales du grand siècle, Paris, Gallimard, 1948), c'est donc ici l'argument de la fermeté virile ( $\left.n^{\circ} 13\right)$, déjà décliné supra $\$ 24$ sous le mode de la «vertu des anciens ».

68. Fam. 5.17.3. Vossius suit la numérotation des Familiares de son époque.

69. Trad. M. Dufresne, op. cit. Cicéron empiète sur l'argument suivant (exposé infra § 29), en passant de "vir » (traduit ici par " homme de courage ») à "sapienter ferres » ("supporter en sage »). Pour d'autres exemples, voir Érasme, De conscribendis..., 49, note 12.

70. L'argument de la fermeté virile met en jeu un pathos, le sentiment de la honte.

71. Après l'exemple des femmes et des amants (supra § 27), voici l'exemple des hommes dans les rites funèbres : la seconde méthode consolatoire est focalisée sur les âmes endeuillées.

72. Voir Plutarque, Consolation à Apollonius sur la mort de son fils, 112f-113a, dans Euvres morales de Plutarque, trad. D. Ricard, Paris, Vve Desaint, 1784, t. II, p. 47 : « [...] Accoutumons notre âme à courir promptement au remède, à réparer le mal, au lieu de nous livrer à des plaintes inutiles. Le législateur des Lyciens [Lycurgue] ordonna que dans le deuil, on prendrait des habits de femme. Il insinuait par là que la tristesse est une passion efféminée qui ne convient pas à des hommes bien nés. C'est la preuve d'un caractère faible et pusillanime que de se livrer à la douleur. » Pour un emploi semblable de cette référence (en liaison avec l'argument de la fermeté virile et avec le pathos de la honte), voir Caussin, De eloquentia sacra..., op. cit., p. 611, item 7 (« Septimo »).

73. Le texte de Cicéron est : « ut et communem ».

74. Le ductus commence ici à apparaître : on passe d'une réflexion philosophique générale sur la nécessité de mourir, à un argument relevant de la morale aristocratique, avant d'en venir à cette vertu pratique et politique par excellence qu'est la prudentia, la sagesse des païens (argument $\mathrm{n}^{\circ} 14$ ) ; et par la prudentia, on bascule du côté de la sagesse chrétienne (thèmes de la vanitas vanitatum et de Dieu propriétaire de nos vies). Comme dans le $\S 27$, où le vocabulaire stoïcien 
côtoyait une référence lyrique antique, la référence épicurienne (Lucrèce) vient ici croiser un thème chrétien.

75. Argument $\mathrm{n}^{\circ} 15 \mathrm{du}$ mépris du monde. Les items énumérés (enfants, richesses...) relèvent de ce que l'on appelait les "biens externes" ou bona externa, d'où ici le «hors de lui », extra se: "J'appelle Biens qu'on trouve dans soi-même, tout ce qui sert à l'embellissement de l'Âme, et à perfectionner le Corps : Et j'appelle Biens étrangers et hors de nous, la Noblesse, les Amis, les Honneurs, et les Richesses." (Aristote, Rhétorique, 1.5, 1360b25, trad. Fr. Cassandre, 1654, graphies modernisées, italiques de Cassandre). À rapprocher d'une injonction qu'Érasme formule sans la développer (De conscribendis..., 49) : le réconfort « sera réalisé [...] si nous minimisons, autant qu'il est possible, le bonheur d'où il [le destinataire] est tombé. ». Voir également Scaliger, Poetices..., III, 122 (repris par Vossius ici même infra § 33) : «[...] on montrera que ce que nous estimons le plus est ce qui a le moins de valeur. Enfin, il faudra emprunter aux philosophes et à ceux qui sont les contempteurs du monde et de la vie tout ce qu'ils disent sur le sujet. "

76. Argument de la dépossession de soi ( $\left.n^{\circ} 16\right)$. Le couple khrèsis / ktèsis (usage / propriété) est en lettres grecques, ici dans le texte des Rhetorices contractae... tout comme dans l'incipit de la lettre de Curius à Cicéron (Fam. 7.29.1, nous traduisons) : "sum enim khrèsei men tuus, ktèsei de Attici nostri. Ergo fructus est tuus, mancipium illius ", "je suis tien par l'usage, mais à Atticus par droit de possession. À toi donc l'usufruit ; à lui la propriété. » La référence une fois encore reste du côté de la sagesse païenne: non pas l'épicurisme (malgré la référence à Lucrèce, qui suit immédiatement), mais le stoïcisme.

Voir par exemple Épictète, Manuel, dans Les Stoïciens, op. cit., 11, p. 1114 : « De rien ne dis jamais : "Je l'ai perdu", mais : "Je l'ai rendu". Ton enfant est-il mort? Il a été rendu. Ta femme est-elle morte ? Elle a été rendue. “On m'a enlevé mon domaine." - Eh bien, lui aussi a été rendu. - "Mais c'est un méchant que celui que me l'a enlevé." - Que t'importe par le moyen de qui le donateur te l'a réclamé ? Tant qu'il te le laisse, prends-en soin comme du bien d'autrui [...]. »

Ou encore Arrien, Entretiens d'Épictète, 4.1, dans Les Stoïciens, ibid., p. 1052-1053 : « Pourquoi donc ai-je reçu tout cela? Pour t'en servir. - Pendant combien de temps? - Tant que celui qui t'a fait ces prêts le voudra. - Mais s'ils me sont nécessaires ? - Ne t'y attache pas et ils ne le seront pas. » Ibid., IV, 1, p. 1055 : «Ainsi le corps nous est-il étranger, étrangères ses parties, étrangère notre fortune; si tu t'attaches à l'une de ces choses comme à ton bien propre, tu subiras le châtiment que mérite celui qui convoite des choses étrangères. "

Voir également Sénèque, De la tranquillité de l'âme, dans Les Stoïciens, ibid., XI, 1 sq. : « Il [le sage] vit comme si on lui avait prêté sa propre existence et comme s'il devait rendre ce prêt sans mécontentement dès qu'on le lui redemandera. [...] La nature qui, la première, nous a ouvert un crédit, peut nous rappeler [...]. »

Sur cette thématique de la dépossession et de l'usage chez les stoïciens, voir T. Bénatouill, «Les possessions du sage et le dépouillement du philosophe», Rursus [En ligne], 3|2008, § 2 («Les modèles juridiques des paradoxes: propriété et usufruit») et $\$ 3$ ("Épictète : l'usage par la dépossession »), URL : http://rursus.revues.org/213.

Ce topos a ensuite été christianisé. Comme le note le même T. Bénatouil (Faire usage : la pratique du stoïcisme, Paris, Vrin, 2007, p. 224, n. 2, avec diverses références, dont Augustin, La Cité de Dieu, 19.10), « la distinction entre usus et fruitio " «sera employée par Saint Augustin afin d'opposer nos rapports aux biens "utiles" de ce monde et au véritable bien moral (honestum) qu'est Dieu. [...] Augustin orientera ainsi la fruitio vers une réalité transcendante. Cet usage néoplatonicien de la fruitio maintiendra voire renforcera son présupposé fondamental (rejeté par les stoïciens): l'homme ne peut trouver un bonheur véritable dans sa seule activité autonome. » Voir aussi Bossuet, Sermons. Le carême du Louvre, Paris, Gallimard, «Folio classique », 2001, "Sermon sur la mort » (22 mars 1662), p. 151 : « Tout nous appelle à la mort : la nature, presque envieuse du bien qu'elle nous a fait, nous déclare souvent et nous fait signifier qu'elle ne peut pas nous laisser longtemps ce peu de matière qu'elle nous prête [...]. » 
77. Note de Vossius : «Lib. 3. » Lucrèce, De rerum natura, trad. H. Clouard, Paris, Garnier, s.d., 3.971.

78. Note de Vossius : «Usu. »

79. Note de Vossius : « Possessione.»

80. Note de Vossius : «I. Cor. 11. $32 »$. Voici le verset 32 dans la traduction d'Érasme du Nouveau Testament (1516) : «At cum iudicamur à Domino, corripimur, ne cum mundo condemnemur » (dans Biblia sacrosancta, éd. H. Bullinger, Zürich, Froshover, 1543, p. 76); texte de la Vulgate : «dum iudicamur autem a Domino corripimur ut non cum hoc mundo damnemur "; traduction de la Bible de Louvain (1550) : «Et quand nous sommes jugés, nous sommes corrigés du Seigneur, afin que ne soyons damnés avec ce monde. »

Une édition des Rhetorices contractae... (Madrid, Antonio de Sancha, 1781, p. 201) corrige la référence en : «I. Cor. II, 13 », soit, dans la traduction d'Érasme, « quae et loquimur, non sermonibus quos docet humana sapientia, sed quos docet ipsus sanctus»; et, dans dans la Bible de Louvain: «lesquelles [choses qui nous sont données de Dieu] aussi nous parlons, non point en paroles doctes de sapience humaine, mais en doctrine d'esprit ». C'est faire référence à la phrase qui précède chez Vossius, avec plusieurs termes qui se répondent : "humana sapientia » pour le N.T., «civili prudentia » pour Vossius ; «docet » pour le N.T., « instructus » pour Vossius ; ou encore « ipsus sanctus » pour le N.T., "Christiana etiam religione » pour Vossius.

Du point de vue sémantique, cette nouvelle référence fait sens, elle souligne que l'important, dans cet alinéa, n'est pas le nouvel argument mentionné (voir note ci-après), mais le fait que c'est le lieu où l'itinéraire tracé par Vossius passe de la " sagesse humaine » à la " religion chrétienne " - et c'est précisément de ce point de basculement (de conversion) dont il est question dans le verset I. Cor. II, 13.

81. On reconnaît ici un argument rapidement effleuré dans les lieux tirés de l'honorable (supra $\S 18)$, l'argument de la Providence $\left(n^{\circ} 7\right)$. Mais la perspective est différente : il ne s'agit pas de qualifier le malheur (un tel malheur est honorable puisque Dieu l'a voulu), mais de continuer à élaborer l'éthos à endosser : après la composante philosophique, une composante proprement chrétienne. La consolation s'engage ici dans la voie de la spiritualité.

82. Il s'agit de l'argument $n^{\circ} 17:$ la vie après la mort (voir la mention du «Christ Sauveur » et la fin de l'énoncé formulaire : «et de ne pas mourir avec le monde »). L'argument met en jeu le pathos de l'espoir. Il est inutile de chercher ce «lieu commun» dans les Loci communes théologiques des Réformés du XVI ${ }^{e}$ siècle (Melanchthon, Wolfgang Musculus...) ou des catholiques (Melchior Cano, De Locis theologicis), qui sont des ouvrages sur les grandes questions de controverse religieuse : aucun n'a de lieu commun intitulé "De prouidentia ", parce que la Providence divine n'est pas un sujet de litige entre catholiques et protestants (mais bien la prédestination, le libre arbitre, etc.).

83. Litt. : «un docteur du christianisme »; doctor se retrouve dans la citation de Caton donnée ensuite, où le mot est alors traduit par «censeur ». En contexte protestant, l'expression fait référence au seul ministère de l'enseignement, là où le ministère du pasteur ajoute une prise en charge fraternelle de la communauté. Docteurs et pasteurs étant souvent associés, nous avons opté pour une traduction qui soit plus largement œcuménique.

84. Le ductus de la conversion est ici récapitulé et trouve son point d'achèvement dans l'argument du devoir d'exemplarité $\left(\mathrm{n}^{\circ} 18\right)$.

85. L'argument du devoir d'exemplarité met a contrario en jeu le pathos de la honte.

86. Distiques moraux de Denys Caton à son fils, 1.30, trad. Levasseur et Chenu, Paris, Garnier Frères, 1864. - On parle aujourd'hui du « Pseudo-Caton».

87. Rappel de la distinction initiale (établie supra $§ 3$ et reprise de Scaliger) entre les malheurs avérés et à venir (voir également supra note 7).

88. La plupart des références ont déjà été données. Nous donnons dans le présent numéro, section ATELIER, les traductions des chapitres de Scaliger et Érasme. La numérotation que 
Vossius donne du chapitre de Ménandre (2.8 au lieu de 2.9, comme indiqué dans les références supra note 2) suit les éditions de la Renaissance (voir par exemple la traduction latine Menandri acutissimi ac sapientiss. rhetoris De genere demonstrativo libri duo. A Natale de comitibus veneto..., Venise, Pietro Boselli, 1559, "De oratione consolatoria», II, 8, p.51b-54b). Pour les lettres consolatoires de Cicéron, nous renvoyons au florilège d'Érasme (voir De conscribendis..., 49 dans ATELIER). Il est à noter que la lettre de consolation adressée à Cicéron pour la mort de sa fille Tullia est de Servius Sixticius (Fam. 4.5) et que la réponse de Cicéron à une consolation suite à ce décès est quant à elle adressée à Servius Sulpicius (Fam. 4.6) : à l'époque de Vossius, on considère que l'ensemble des Fam. 4.1-6 sont adressées à (ou écrites par) un seul et même personnage, Servius Sulpicius (le nom de Sixticius n'apparaît pas dans Fam.4.5, laquelle a seulement "Servius»). Outre la Consolation à Apollonius, Vossius ajoute pour Plutarque la Consolation à sa femme sur la mort de leur fille; et d'Aelius Aristide, un discours qui n'est pas une consolation mais à nouveau un épicède (comme pour la poésie de Stace citée supra \$27), le Discours funèbre en l'honneur d'Étéonée (Or. 31). La présentation de cette référence sous l'angle de la consolation se trouve dans les mêmes termes chez Caussin (De eloquentia sacra..., op. cit., p. 611 : «Aristidis ad Eteoni parentes, consolatio »).

89. Vossius reprend ici le développement de Scaliger (Poetices..., III, 122): d'abord en le paraphrasant (du début du premier alinéa à « devra être divisée en deux parties »; puis en le citant littéralement: de "Dans la première, on dira " jusqu'à la fin de l'avant-dernier alinéa. L'imminence du danger est l'un des tout premiers lieux de la peur: Aristote, Rhétorique, 2.5, $1382 \mathrm{a} 25$, op. cit., p. 288 : « et cela s'ils [les maux] paraissent non pas éloignés mais d'une proximité imminente. "

90. Dans le même chapitre d'Aristote, la peur des puissants est un des lieux suivants, peu après celui sur l'imminence du danger et le précisant. Aristote, Rhétorique, 2.5, 1382a30, op. cit., p. 289 : « même les signes avant-coureurs de ces choses sont effrayants [...] telles la haine et la colère de personnes qui ont le pouvoir de faire du mal ». L'exemple du tyran invite à recentrer les consolations pour la crainte d'un malheur, sur les craintes politiques (menaces de procès, d'exil, d'indignité, de condamnation à mort...).

91. Poetices..., III, 122 dans l'édition L. Deitz, op. cit., p. 198.

92. Récriture de Vossius, qui adapte le propos de Scaliger au cas de l'oratio. On se souvient en effet que le classement de Scaliger n'opère pas sur les discours mais sur les poèmes, et en l'occurrence sur les silves, d'où sa formulation : « [...] il faudra diviser le poème en deux parties. » 93. Scaliger organise la progression en fonction du pathos, d'abord l'espoir puis l'assurance. Concernant l'espoir, nous retrouvons l'argument de la conjecture $\left(\mathrm{n}^{\circ} 1\right)$, convoqué initialement pour les maux remédiables (supra § 7) ainsi que pour la circonstance du temps (supra § 16).

94. Réconfort par atténuation du malheur : avec mention de l'argument 15 (le mépris du monde). 95. Allusion à tous les ouvrages qui ont pour titre De contemptu mundi (Le mépris du monde), dans la tradition stoïcienne et chrétienne.

96. Litt. : « aux nombres ». Pour reprendre une suggestion de Francis Goyet, les numeri sont les rythmes de la prose d'art latine, à la fois proches et distincts du rythme des vers; et ce sont eux qui différencient la prose d'art de la prose de tous les jours. Voir Cicéron, Orator, 174 sq.; Quintilien, Institutio oratoria, 9.4.45-46.

97. Parce qu'il rattache l'efficacité de la consolation au movere, Scaliger soulève ici l'objection des banalités (ce que nous appelons aujourd'hui lieux communs) : comment leur redonner force et énergie ? La réponse semble contournée : en recourant aux figures de mots et de rythmes. Il manque ici un troisième terme, qui permet d'expliciter le lien : c'est celui de l'amplification. Depuis Cicéron en effet, l'amplification est liée d'une part aux effets pathétiques, et d'autre part aux ressources de l'elocutio (à l'art de l'éloquence). Voir Cicéron, Partitions oratoires, 19.67 (trad. M. Bompart, 1835) : « faire naître ou [..] exalter les passions, c'est le but de l'amplification. » Pour une reprise tardive, voir par exemple J. Jouvancy, s.j., Candidatus rhetoricae (Paris, Barbou, 1712, 
p. 68), trad. H. Ferté, L'élève de rhétorique (Paris, Hachette, 1892, p. 79) : « Avec Cicéron, j'entends par amplification une affirmation plus forte que d'ordinaire qui, grâce à l'émotion qu'excite l'orateur, entraîne la persuasion [...]. Quand on veut émouvoir les auditeurs, on emploie des amplifications plus importantes, et c'est en cela que triomphe l'éloquence. »

98. Scaliger reprend ici la distinction d'Érasme entre les âmes faibles et les âmes fortes (voir De conscribendis..., 49 et supra note 24), pour en tirer deux stratégies différentes de réconfort : pour les âmes faibles (ci-avant), une stratégie d'exhortation jouant sur les ressorts de l'espoir ou de l'assurance et animant le propos par l'amplification figurale ; pour les âmes fortes (ci-après), une stratégie de renforcement par insinuation, appuyée sur des exemples de constances (argument $\mathrm{n}^{\circ} 12$ ) et sur le pathos de l'émulation (ou son envers, la honte, si les exemples sont sans effet).

99. Scaliger emploie fides, mot par lequel Cicéron définit le but de la rhétorique (« fidem facere »).

100. Vossius a omis l'adjectif frequentia présent chez Scaliger (Poetices..., III, 122): «quam exemplorum frequentia persuasio insinuanda ».

101. Si l'on tente une récapitulation des sources où puiser pour raisonner l'âme éplorée et la (ré)conforter, les arguments répertoriés reviennent parfois d'une section à l'autre, produisant un brouillage entre les différentes méthodes (d'autant plus que s'y ajoute l'ensemble des raisons visant au préalable à la captatio benevolentiae, c'est-à-dire mobilisant l'amor). Au terme du chapitre, il est cependant possible de résumer les raisons du réconfort proprement dit en deux grands ensembles, les raisons pathétiques et les raisons éthiques :

\section{RAISONS DU RÉCONFORT : SYNTHÈSE}

\section{Raisons pathétiques : espoir et honte}

Pour l'espoir (associé à la confidentia, à l'assurance) : voir les arguments $n^{\circ} 1$ (La conjecture : $\S 7,16,33)-n^{\circ} 5$ (Les suites heureuses : § 16) - $n^{\circ} 17$ (La vie après la mort : § 30).

Pour la honte (associée à l'émulation) : voir les arguments $n^{\circ} 6$ (Les malheurs plus grands : § 16) - $\mathrm{n}^{\circ} 12$ (Les exemples de constance : § 23,33) - $\mathrm{n}^{\circ} 13$ (La fermeté virile : § 24, 28) - $\mathrm{n}^{\circ} 18$ (Le devoir d'exemplarité : § 31).

\section{Raisons éthiques : vertu et sagesse}

Appel à la vertu : voir les arguments $n^{\circ} 3$ (La bonne conscience : $\left.\$ 10,18\right)-n^{\circ} 8$ (La réputation accrue par le malheur: $\$ 19,24)-n^{\circ} 9$ (La bonification par l'épreuve : 19$)-n^{\circ} 10$ (Le souvenir futur du malheur présent : § 21).

Appel à la sagesse (philosophique et chrétienne) : voir les arguments $\mathrm{n}^{\circ} 2$ (La nécessité de souffrir et de mourir: § 10,27) - $\mathrm{n}^{\circ} 4$ (La patience face aux épreuves : § 15, 22, 24) - $\mathrm{n}^{\circ} 7$ (La Providence ou la volonté de Dieu : § 18,30) - n॰ 10 (L'irritation du mal par les pleurs : § 20,24) $\mathrm{n}^{\circ} 14$ (La sagesse prudentielle: §29) - $\mathrm{n}^{\circ} 15$ (Le mépris du monde: §29,33) - n 16 (La dépossession de soi : § 29).

\section{AUTEURS}

\section{G. J. Vossius}

Gerhard Johann Voss, 1577-1649 